\title{
A Physiologically Based Pharmacokinetic Drug-Disease Model to Predict Carvedilol Exposure in Adult and Paediatric Heart Failure Patients by Incorporating Pathophysiological Changes in Hepatic and Renal Blood Flows
}

\author{
Muhammad Fawad Rasool • Feras Khalil • \\ Stephanie Läer
}

Published online: 14 March 2015

(c) The Author(s) 2015. This article is published with open access at Springerlink.com

\begin{abstract}
Background and Objective Chronic diseases are associated with pathophysiological changes that could have profound impacts on drug pharmacokinetic behaviour, with a potential need to modify the administered drug therapy. It is important to acknowledge that most patients with chronic illnesses do not have a single, predominant condition but suffer from multiple comorbidities. The rapid advancement in physiologically based pharmacokinetic (PBPK) modelling, as well as the increasing quantitative knowledge of disease-related pathophysiological changes, facilitate building of drug-disease models. However, there are only a few published examples of PBPK models incorporating the pathophysiological changes that occur with chronic diseases. The objective of this study was to develop PBPK models that incorporate the haemodynamic changes in hepatic and renal blood flows occurring in chronic heart failure (CHF) and to evaluate these changes in adults and children, using carvedilol as a model drug.

Methods After a comprehensive literature search to select the model input parameters, two PBPK models were
\end{abstract}

M. F. Rasool and F. Khalil contributed equally to this work.

Electronic supplementary material The online version of this article (doi:10.1007/s40262-015-0253-7) contains supplementary material, which is available to authorized users.

M. F. Rasool · F. Khalil · S. Läer

Department of Clinical Pharmacy and Pharmacotherapy,

Heinrich-Heine University, 40225 Düsseldorf, Germany

M. F. Rasool ( $\square)$

Faculty of Pharmacy, Bahauddin Zakariya University,

Multan 60800, Pakistan

e-mail: fawadrasool@bzu.edu.pk;

fawad.rasool@uni-duesseldorf.de developed. Model 1 was based on human liver and intestinal microsome clearances, and model 2 was based on clearance by specific cytochrome P450 enzymes. After evaluation of both models in healthy adults, the reduced hepatic and renal blood flows were incorporated into the developed models to predict carvedilol exposure in the adult $\mathrm{CHF}$ population. The adult carvedilol models were scaled down to children by using Simcyp ${ }^{\circledR}$ (Simcyp Ltd, Sheffield, UK). In order to show the impact of reduced organ blood flows on carvedilol disposition, the predictions in the CHF population were made with and without reductions in organ blood flows.

Results The predictions made by both models in healthy adults were comparable and within the 2-fold error range. In adults with $\mathrm{CHF}$, the mean observed/predicted ratio $\left[\mathrm{ratio}_{(\mathrm{Obs} / \mathrm{Pred})}\right]$ for oral clearance $(\mathrm{CL} / F)$ without reductions in organ blood flows was outside the 2-fold error range, i.e. 0.34 (95\% confidence interval [CI] 0.31-0.37), with use of both models. The mean $\mathrm{CL} / F$ ratio $(\mathrm{Obs} / \mathrm{Pred})$ values after incorporation of reduced organ blood flows were 1.0 (95\% CI 0.92-1.08) and 0.95 (95\% CI 0.88-1.03) with use of models 1 and 2, respectively. The mean ratio $(\mathrm{Obs} /$ Pred) values for the pharmacokinetic parameters were not improved after incorporation of reduced blood flows in paediatric patients, except in those above 17 years of age, who were categorized according to the New York Heart Association classification of $\mathrm{CHF}$, where the CL/F ratio $_{(\mathrm{Obs} / \mathrm{Pred})}$ values in two patients were closer to unity. Conclusion There was a strong connection between a decrease in hepatic clearance of carvedilol and an increase in the severity of $\mathrm{CHF}$, especially in adults and in paediatric patients above 17 years of age. The incorporated reductions in hepatic and renal blood flows occurring in moderate and severe $\mathrm{CHF}$ patients resulted in improved predictions of carvedilol exposure. The developed models 
can be extended to predict exposures of drugs with high hepatic extraction in the CHF population.

\section{Key Points}

Reductions in hepatic blood flow can significantly affect the first-pass and systemic hepatic metabolism, the bioavailability, and thus the total exposure of drugs with high hepatic extraction ratios. For oral administration, the total effect of these changes on the exposure is more complex, since any changes in reduced systemic clearance $(\mathrm{CL} / F)$ will be compensated for by reduced bioavailability (expected from higher hepatic extraction during the first pass).

In chronic heart failure (CHF), a decrease in the CL/ $F$ of carvedilol can be modelled by incorporation of hepatic and renal blood flow changes occurring in this disease, using a physiologically based pharmacokinetic (PBPK) drug-disease model.

The incorporation of reduced hepatic and renal blood flows into the presented PBPK models caused a significant improvement in prediction of carvedilol exposure in adult CHF patients, but with regard to paediatric predictions, the improvements were seen only in adolescents categorized according to the New York Heart Association classification of CHF with available pharmacokinetic data sets.

\section{Introduction}

Chronic diseases are associated with pathophysiological changes that could have profound impacts on the pharmacokinetic behaviour of drugs, with a potential need to modify the administered drug therapy [1]. It is important to acknowledge that most patients with chronic illnesses do not have a single, predominant condition but suffer from multiple comorbidities. The rapid advancement in physiologically based pharmacokinetic (PBPK) modelling, as well as the increasing quantitative knowledge of diseaserelated pathophysiological changes, facilitate building of drug-disease models that can incorporate these changes to predict their pharmacokinetic impact [2-8]. A PBPK model that is modified according to the pathophysiology of a disease could be extended, after its evaluation, to a wide variety of drugs because of its mechanistic nature. An additional gain can be further obtained if an extrapolation from adult patients to special populations, e.g. children, occurs.
However, despite the clinical significance, there are only a few published examples of PBPK models incorporating pathophysiological changes occurring with chronic diseases [2-6]. Interestingly, to date, there has been no published report of a drug-disease PBPK model evaluated for predicting drug exposure in chronic heart failure (CHF) adult and paediatric patients after incorporation of reductions in organ blood flows, despite the high prevalence and the clinical importance of this disease. Instead, there are some published PBPK models that have explored drug pharmacokinetics in liver cirrhosis [2, 6], in chronic kidney disease $[3,5,8]$ and in patients with low cardiac output syndrome undergoing cardiac surgery [4]. In CHF, there is a gradual decrease in hepatic blood flow $\left(Q_{\mathrm{H}}\right)$ with increasing severity of the disease, which has previously been quantified in adults [9]. Development and evaluation of a PBPK model that can incorporate the relevant hemodynamic changes seen in CHF and that can quantify the impact of those change could be of great use. This is particularly true for drugs with high hepatic extraction ratios, as these are most vulnerable to $Q_{\mathrm{H}}$ changes, resulting in a significant effect on their clearance [10]. Under intravenous administration, the change in clearance will lead to a direct change in drug exposure, while in the case of oral administration, the effect of clearance changes on exposure is more complex, as it is confounded and partly compensated by the change in bioavailability.

In light of this information, and in order to evaluate a PBPK CHF model with incorporated organ blood flow changes, a model drug being used clinically in CHF with high hepatic extraction was searched. Carvedilol, a thirdgeneration beta-blocker used in the treatment of various cardiovascular disorders, undergoes extensive first-pass metabolism with a high hepatic extraction ratio $(0.7)$ and absolute oral bioavailability of 22-24\% [11-14]. Because of its high hepatic extraction and the availability of observed clinical data in both adult and paediatric populations [13, 15-18], carvedilol could serve as a model drug to demonstrate if a PBPK disease model could accurately predict the pharmacokinetic changes occurring in CHF.

The main objective of this study was to develop PBPK models that incorporate the hemodynamic changes in hepatic and renal blood flows occurring in CHF, and to evaluate it in adults and children, using carvedilol as a model drug.

\section{Methods}

\subsection{Modelling Software and Strategy for Model- Building}

Simcyp ${ }^{\circledR}$ Simulator version 13.1 (Simcyp Ltd, Sheffield, UK) was used to provide the general structure of the 
developed PBPK models. Simcyp ${ }^{\circledR}$ is a population-based PBPK simulator used for bottom-up mechanistic drug modelling of absorption, distribution, metabolism and elimination (ADME). This software combines in vitro and in vivo data on drug absorption, distribution and elimination with the physicochemical properties of the drug, in addition to incorporating anatomical and physiological data, to simulate the systemic exposure to the drug in virtual healthy and diseased populations [19].

In the presented work, two PBPK models that differed only in terms of the assigned clearance inputs were developed: model 1 , with in vitro intrinsic clearance $\left(\mathrm{CL}_{\mathrm{int}}\right)$ values based on human liver and intestinal microsomes (HLM and HIM); and model 2, based on cytochrome P450 (CYP) enzyme clearances. The choice of presenting both models was made because fewer assumptions were made with model 1; however, model 2 can offer an additional gain of information in the future by allowing the user to incorporate information on genetic polymorphisms of the metabolizing enzymes.

A systematic approach was used in developing the PBPK models, starting by screening and extracting drugspecific input parameters and clinical pharmacokinetic data from the published literature, followed by the incorporation of these data into the software and the selection of the underlying physiological models and final model parameters, and ending with the final evaluation of model predictions of drug disposition and absorption. The modelbuilding was done in a stepwise strategy similar to that reported previously [20]. In this strategy, intravenous drug application in healthy adult subjects is first simulated to avoid the complexities of the oral absorption process so that a wide range of drug-dependent parameters that govern the drug disposition, including clearance inputs and the percentage CYP enzyme contributions, are chosen or optimized. For oral application, parameters from the previous step are kept, and additional parameters that control and influence drug absorption, such as intestinal permeability, are also included. In order to do so, one third $(n=3$ : one intravenous and two oral) of the collected observed pharmacokinetic data in adults were used to optimize the model parameterization, two thirds $(n=6)$ were used later for a subsequent model verification, and all data were used in the final model evaluation. Changes of hepatic and renal blood flows observed in CHF were then incorporated into this adult model to predict carvedilol pharmacokinetics in CHF patients. At the end, the final adult CHF model was then scaled down to children, using the paediatric module of Simcyp ${ }^{\circledR}$. In the adult and paediatric CHF populations, predictions were made in duplicate, using both models, i.e. with and without incorporation of the reduced organ blood flows. The workflow for the development of the PBPK models is shown in Fig. 1.

\subsection{Model Structure and Parameterization}

A comprehensive literature search was performed to extract necessary in vivo and in vitro ADME data to complete the parameterization of the models. These data include, for example, the drug-specific physicochemical properties, drug clearance information and measures of drug intestinal permeability. The final set of model input parameters is summarized in Table 1. The two developed models were similar in terms of all of the input parameters, except for the method used for assigning clearance. A detailed description of the various model components with their parameterization is given below.

\subsubsection{Absorption}

The advanced dissolution, absorption and metabolism (ADAM) model with the default values of fasting gastric emptying time (mean $0.4 \mathrm{~h}$ with a coefficient of variation [CV] of $38 \%$ ) and small intestinal transit time (mean $3.33 \mathrm{~h}$ with Weibull distribution, $\alpha=2.92$ and $\beta=4.04$ ) was used to predict drug absorption [21]. The predicted human jejunum permeability $\left(P_{\text {eff,man }}\right)$ of carvedilol was $1.94 \times 10^{-4} \mathrm{~cm} / \mathrm{s}$, which was obtained using in vitro $\mathrm{Ca}$ co-2 permeability $\left(P_{\text {app }}\right)$ data calibrated with reference values for propranolol and metoprolol [22]. In addition, the predicted absorbed fraction of carvedilol was 0.896 , which is consistent with carvedilol being a highly permeable drug belonging to Biopharmaceutics Classification System (BCS) class II.

The $P_{\text {eff,man }}$ value was increased from $1.94 \times 10^{-4}$ to $7.4 \times 10^{-4} \mathrm{~cm} / \mathrm{s}$ in all simulations of adult and paediatric subjects whenever carvedilol was administered as an oral suspension, because of the observed increase in carvedilol bioavailability. This optimization was achieved by comparison of the predicted pharmacokinetic parameters in healthy adults with the observed data, after administration of a $50 \mathrm{mg}$ oral capsule and suspension [18]. Although fast gastric emptying of liquid dosage formulations has already been reported and can, theoretically, be a parameter to adjust [23], it was not possible to account for the previously mentioned increase in drug absorption by only reducing the gastric emptying time.

With regard to active transport, there are some reports suggesting a possible role of P-glycoprotein (P-gp) in carvedilol disposition [17, 24]. However, the International Transporter Consortium (ITC) has stated in its guidelines and established decision trees that, when the net flux ratio of a drug in a bidirectional transport assay, such as Caco-2, is less than 2, then it is a poor or non-P-gp substrate [25], which was the case with carvedilol (its net flux ratio is 1.3 [22]). This suggests that carvedilol is a poor substrate for P-gp, which is in harmony with reports stating that 


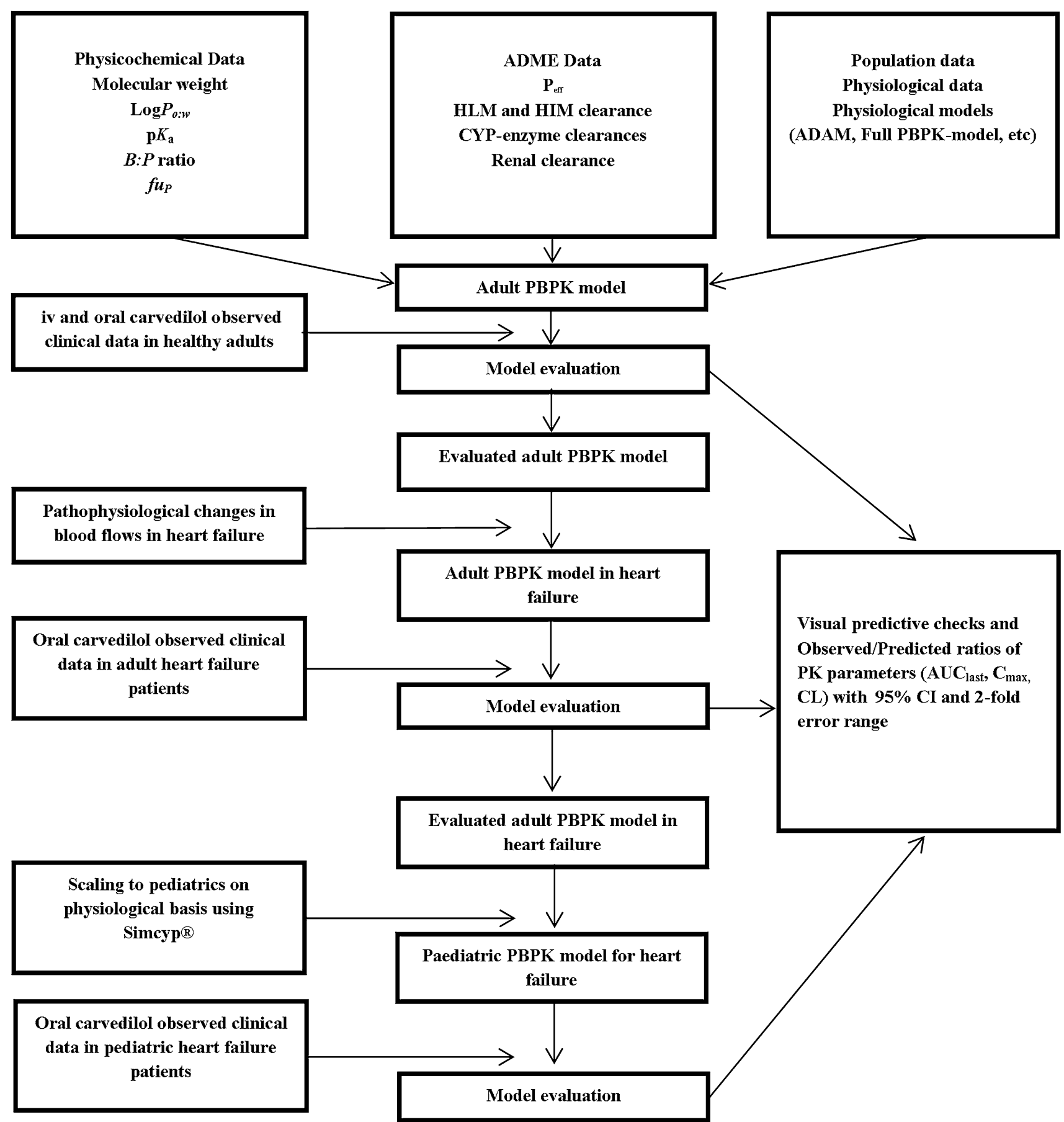

Fig. 1 Workflow for the development of adult and paediatric physiologically based pharmacokinetic (PBPK) carvedilol heart failure models. ADAM advanced dissolution, absorption and metabolism, $A D M E$ absorption, distribution, metabolism and elimination, $A U C_{\text {last }}$ area under the systemic drug concentration-time curve from time zero to the time of the last measured concentration,

carvedilol is a strong P-gp inhibitor but not a good substrate [26]. In light of the aforementioned information, no P-gp-specific data were incorporated into the developed PBPK models.
$C I$ confidence interval, $C L$ clearance, $C_{\max }$ maximum concentration, $C Y P$ cytochrome $\mathrm{P} 450, f u_{P}$ fraction of unbound drug in plasma, $H I M$ human intestinal microsomes, HLM human liver microsomes, $i v$ intravenous, $\log P_{o: w}$ octonal-water partition coefficient, $P_{\text {eff.- }}$ man human jejunum permeability, $P K$ pharmacokinetic, $p K_{a}$ acid dissociation constant

\subsubsection{Distribution}

The full PBPK model for the prediction of drug distribution was used in both developed models. The drug volume of 
Table 1 Main drug-dependent parameters and characteristics of the presented physiologically based pharmacokinetic (PBPK) models

\begin{tabular}{|c|c|c|c|c|}
\hline Parameter & Model 1 & Model 2 & Reference value & References \\
\hline Molecular weight $(\mathrm{g} / \mathrm{mol})$ & 406.47 & 406.47 & 406.47 & PubChem \\
\hline $\log P_{\mathrm{o}: \mathrm{w}}$ & 4.19 & 4.19 & 4.19 & PubChem \\
\hline $\mathrm{p} K_{\mathrm{a}}$ & 7.97 & 7.97 & 7.97 & {$[55]$} \\
\hline \multicolumn{5}{|l|}{ Absorption } \\
\hline Model & ADAM & & & \\
\hline$P_{\text {eff,man }}(\mathrm{cm} / \mathrm{s})$ in tablets and capsules ${ }^{\mathrm{a}}$ & $1.94 \times 10^{-4}$ & $1.94 \times 10^{-4}$ & $1.94 \times 10^{-4}$ & {$[22]$} \\
\hline$P_{\text {eff,man }}(\mathrm{cm} / \mathrm{s})$ in oral suspension ${ }^{\mathrm{b}}$ & $7.4 \times 10^{-4}$ & $7.4 \times 10^{-4}$ & - & - \\
\hline \multicolumn{5}{|l|}{ Distribution } \\
\hline Model & \multicolumn{4}{|l|}{ Full PBPK } \\
\hline Prediction method ${ }^{\mathrm{c}}$ & \multicolumn{4}{|c|}{ Paulin and Theil method } \\
\hline $\mathrm{B}: \mathrm{P}$ ratio & 0.69 & 0.69 & 0.69 & {$[56]$} \\
\hline $\mathrm{fu}_{\mathrm{P}}$ & 0.0054 & 0.0054 & 0.0054 & {$[56]$} \\
\hline \multicolumn{5}{|l|}{ Elimination } \\
\hline $\mathrm{CL}_{\mathrm{iv}}(\mathrm{L} / \mathrm{h})$ & - & 38 & $35.34^{\mathrm{d}}$ & {$[18]$} \\
\hline HLM CL $\mathrm{C}_{\text {int }}(\mu \mathrm{L} / \mathrm{min} / \mathrm{mg}$ protein $)$ & 246 & - & 246 & {$[28]$} \\
\hline Fraction unbound in microsomal incubation & 0.04 & - & 0.04 & {$[28]$} \\
\hline HIM $\mathrm{CL}_{\text {int }}(\mu \mathrm{L} / \mathrm{min} / \mathrm{mg}$ protein $)$ & 11.4 & - & 11.4 & [29] \\
\hline CYP2D6 CL $\mathrm{L}_{\text {int }}(\mu \mathrm{L} / \mathrm{min} / \mathrm{mg} / \mathrm{pmol} \text { of isoform })^{\mathrm{e}}$ & - & 339.7 & - & - \\
\hline CYP1A2 $\mathrm{CL}_{\text {int }}(\mu \mathrm{L} / \mathrm{min} / \mathrm{mg} / \mathrm{pmol} \text { of isoform })^{\mathrm{e}}$ & - & 8.71 & - & - \\
\hline CYP2C9 $\mathrm{CL}_{\text {int }}(\mu \mathrm{L} / \mathrm{min} / \mathrm{mg} / \mathrm{pmol} \text { of isoform })^{\mathrm{e}}$ & - & 3.1 & - & - \\
\hline CYP2E1 CL int $(\mu \mathrm{L} / \mathrm{min} / \mathrm{mg} / \mathrm{pmol} \text { of isoform })^{\mathrm{e}}$ & - & 3.71 & - & - \\
\hline Additional HLM clearance $(\mu \mathrm{L} / \mathrm{min} / \mathrm{mg} \text { protein })^{\mathrm{e}}$ & - & 906.7 & - & - \\
\hline $\mathrm{CL}_{\mathrm{R}}(\mathrm{L} / \mathrm{h})$ & 0.25 & 0.25 & 0.25 & {$[34]$} \\
\hline
\end{tabular}

ADAM advanced dissolution, absorption and metabolism, $B: P$ blood-to-plasma, $C L_{\text {int }}$ intrinsic clearance, $C L_{i v}$ intravenous clearance, $C L_{R}$ renal clearance, $C Y P$ cytochrome P450, $f u_{P}$ fraction of unbound drug in plasma, $H I M$ human intestinal microsomes, $H L M$ human liver microsomes, $K_{p}$ tissue-to-plasma partition coefficient, $\log P_{o: w}$ octonal-water partition coefficient, $P_{a p p}$ in vitro permeability, $P_{\text {eff,man }}$ human jejunum permeability, $p K_{a}$ acid dissociation constant, $V_{s s}$ drug volume of distribution at steady state

${ }^{\text {a }} P_{\text {eff,man }}$ calculated from the $P_{\text {app }}$ value of a Caco-2 assay by calibration with metoprolol and propranolol, using Simcyp ${ }^{\circledR}$

${ }^{\text {b }}$ Optimized $P_{\text {eff,man }}$

c Paulin and Theil method with the Berezhkovskiy correction, as the prediction method for $V_{\mathrm{ss}}$ and $K_{\mathrm{p}}$ (predicted $V_{\mathrm{ss}} 1.69 \mathrm{~L} / \mathrm{kg}$ )

${ }^{\mathrm{d}} \mathrm{CL}_{\text {iv }}$ range $22.5-50.8 \mathrm{~L} / \mathrm{h}$

e Values calculated by using the retrograde model in Simcyp ${ }^{\circledR}$

distribution at steady state $\left(V_{\mathrm{ss}}\right)$ values and the specific tissue-to-plasma partition coefficient $\left(K_{\mathrm{p}}\right)$ values were calculated by the Paulin and Theil method with the Berezhkovskiy correction [27]. As a result, the predicted $V_{\text {ss }}$ value of $1.69 \mathrm{~L} / \mathrm{kg}$ by this method was comparable to the reported value of $1.62 \mathrm{~L} / \mathrm{kg}^{1}$ in the literature [18].

\subsubsection{Elimination}

The human liver and human intestinal microsome $\mathrm{CL}_{\mathrm{int}}$ values were used for the prediction of drug clearance in model 1 [28, 29]. Model 2 used CYP enzyme $\mathrm{CL}_{\text {int }}$

$1 V_{\text {ss }}$ was calculated by using non-compartmental analysis in Phoenix WinNonLin ${ }^{\circledR}$ software (Pharsight Corporation, CA, USA). values calculated by the retrograde model for enzyme kinetics in Simcyp ${ }^{\circledR}$. The retrograde model is one of the models incorporated in Simcyp ${ }^{\circledR}$ that allows the calculation of $\mathrm{CL}_{\text {int }}$ values of the relevant metabolizing enzymes from in vivo measured clearance. A detailed description of this model has already been published elsewhere [30, 31]. In brief, the retrograde model utilizes an in vivo measure of the adult intravenous or oral clearance with known fractions of hepatic clearance $\left(\mathrm{CL}_{\mathrm{H}}\right)$, renal clearance $\left(\mathrm{CL}_{\mathrm{R}}\right)$ or any additional clearance, the unbound drug fraction in plasma $\left(\mathrm{fu}_{\mathrm{p}}\right)$, the blood-to-plasma ratio of the drug concentrations, and $Q_{\mathrm{H}}$ to calculate total hepatic $\mathrm{CL}_{\text {int }}$ as given in the following equation of the well-stirred liver model (Eq. 1):

$\mathrm{CL}_{\mathrm{int}}=\frac{Q_{\mathrm{H}} \times \mathrm{CL}_{\mathrm{H}}}{\mathrm{fu}_{\mathrm{B}} \times\left(Q_{\mathrm{H}}-\mathrm{CL}_{\mathrm{H}}\right)}$ 
In a second step, this hepatic $\mathrm{CL}_{\mathrm{int}}$ value is divided between the involved hepatic elimination pathways (i.e. the different CYP enzymes), using input information about the percentage contribution of these metabolizing enzymes in drug clearance.

The contributions of the CYP enzymes that are involved in carvedilol metabolism were guided by the available evidence in the literature. It has been reported that CYP2D6 is the major contributing CYP enzyme, while CYP1A2, CYP2C9, CYP2E1 and CYP3A4 are involved with a minor role $[17,32,33]$. In the literature, there is no clear information about the relative contributions of the CYP enzymes to the total carvedilol clearance, except for that of CYP2D6 (50-62 \% [17, 33]). From the remaining CYP enzymes believed to be involved in the metabolic pathways of carvedilol, CYP1A2, CYP2C9 and CYP2E1 were reported to be of further importance [32]. In light of the aforementioned information and the fact that the relative amount of carvedilol glucuronidation is around $22 \%$ [14], $80 \%$ of total carvedilol clearance was assigned to the following CYP enzymes-60 \% CYP2D6, $10 \%$ CYP1A2, $5 \%$ CYP2C9 and $5 \%$ CYP2E1 - and the remaining $20 \%$ was assigned to glucuronidation as additional clearance. A $\mathrm{CL}_{\mathrm{R}}$ value of $0.25 \mathrm{~L} / \mathrm{h}$ was used in both models 1 and 2 [34].

Three different uridine diphosphate glucuronosyltransferase (UGT) enzymes are believed to be involved in the metabolism of carvedilol: UGT1A1, UGT2B4 and UGT2B7. In the literature, there is no clear information about their individual relative contributions to the metabolic clearance of carvedilol; therefore, the collective impact of these UGT enzymes was reflected in an assigned additional clearance, which constituted about $20 \%$ of the total carvedilol clearance. This is in a good agreement with the reported values of 20-23\% in healthy adults [14].

In model 1, a CV of $30 \%$ was assigned to HIM and HLM CL $\mathrm{Cin}_{\text {int }}$ In model 2, a CV of $30 \%$ was assigned to the additional clearance, whereas for the CYP enzyme CL $\mathrm{L}_{\text {int }}$ values obtained by the retrograde model, the observed variability was due to variations in the physiological and anatomical variables, such as liver weight, microsomal proteins per gram of liver, abundance of each specific CYP enzyme, cardiac output and $Q_{\mathrm{H}}$.

$\mathrm{CL}_{\mathrm{H}}$ was predicted from the well-stirred liver model [35], using Eq. 2:

$\mathrm{CL}_{\mathrm{H}}=\frac{Q_{\mathrm{H}} \times \mathrm{fu}_{\mathrm{B}} \times \mathrm{CLu}_{\mathrm{H}, \text { int }}}{Q_{\mathrm{H}}+\mathrm{fu}_{\mathrm{B}} \times \mathrm{CLu}_{\mathrm{H}, \text { int }}}$

The $Q_{\mathrm{H}}$ changes occurring in $\mathrm{CHF}$ were incorporated into the model for prediction of the disposition of carvedilol in patients with $\mathrm{CHF}$.

The oral bioavailability $(F)$ was predicted by using Eq. 3:
$F=F_{\mathrm{a}} \times F_{\mathrm{g}} \times F_{\mathrm{h}}$

where $F_{\mathrm{a}}$ is the fraction of the drug absorbed, $F_{\mathrm{g}}$ is the fraction escaping metabolism in the gastrointestinal tract and $F_{\mathrm{h}}$ is the fraction escaping hepatic metabolism.

\subsection{Model Scaling to Children}

Only when the adult CHF models were shown to predict carvedilol pharmacokinetics adequately were they scaled to the children by using the paediatric module of Simcyp ${ }^{\circledR}$. This module of the program incorporates a wide variety of age-specific physiological and anatomical parameters, which facilitates the paediatric scaling of drug clearance on a physiological basis. Because of the use of an oral suspension of carvedilol in both paediatric clinical trials [15], an optimized value of $P_{\text {eff,man }}$ was used in all paediatric predictions, as in the adults who were administered the oral suspension of carvedilol. The paediatric module of the program also accounts for the age-related changes occurring in the total body composition, when predicting distribution of drugs [36]. The key determinants for predicting drug clearance in the paediatric module are the age-specific changes occurring in plasma protein binding, blood volume, organ blood flows, organ sizes and ontogeny, and the abundance of hepatic CYP enzymes [36]. In model 2, the ontogeny profiles of the CYP enzymes were incorporated by default within the program, but in model 1 , in order to create an ontogeny profile similar to that of model 2 , a customized ontogeny profile was created by addition of the fractional contributions of the CYP enzymes with their respective ontogenies [32, 33, 37]. All of the paediatric patients were diagnosed with $\mathrm{CHF}$, and the reductions in hepatic and renal blood flows were incorporated into the paediatric models by categorization of the patients into different CHF categories, according to their modified Ross scores [38].

\subsection{Changes in Blood Flow to Eliminating Organs in Heart Failure}

The liver and kidney are the main organs of drug elimination from the human body; therefore, changes in the blood flows to these organs are of pharmacokinetic importance. In adult heart failure patients, the reduction in $Q_{\mathrm{H}}$ is known to increase with increased severity of the disease [9]. In brief, these organ blood flow reductions occurring in adult heart failure patients were quantified after measurement of the hepatic and renal plasma flows in healthy and heart failure patients by administration of doses of intravenous indocyanine green and intravenous $p$-aminohippurate and measurement of their respective clearances [9]. The quantified reductions were $23.5,46.2$ and $53.7 \%$ of 
normal $Q_{\mathrm{H}}$ in mild, moderate and severe CHF patients, respectively [9]. Moreover, the reduction in renal blood flow was also shown to be greater with increased severity of CHF; however, this reduction is not absolutely linear when we move from moderate to severe $\mathrm{CHF}$, as it has been reported to be around 22.2, 45 and $37.1 \%$ of normal blood flow in mild, moderate and severe CHF patients, respectively [9].

In adult patients, the New York Heart Association (NYHA) functional classification system provides a simple way of classifying the severity of CHF [39], where, for example, NYHA class II reflects mild CHF and class III reflects moderate CHF, whereas class IV stands for severe CHF [39]. As a result, the reported reductions in blood flows to the liver and the kidney could be directly correlated in adults with the NYHA classification. In order to predict drug exposure in CHF patients, using a PBPK model, these reductions in organ blood flows were assigned to the simulated virtual populations within Simcyp ${ }^{\circledR}$ by reduction of the percentage blood flows to the liver and kidney, respectively. When the drug exposure in a population comprising NYHA class III (moderate CHF) and class IV (severe CHF) patients had to be predicted, the mean organ blood flow reductions in moderate and severe $\mathrm{CHF}$ from the reported values were used [9], i.e. reductions of 49.9 and $41 \%$ of the normal hepatic and renal blood flows, respectively.

In paediatric CHF patients, because of the difficulty in assessing physical activity, the NYHA classification is not used to assess the severity of the CHF; instead, the Ross score is the system commonly used in children [40]. According to the Ross score, a score of 0-2 categorizes the patient as asymptomatic, 3-6 as having mild CHF, 7-9 as having moderate $\mathrm{CHF}$ and $10-12$ as having severe $\mathrm{CHF}$ $[38,40]$. As there were no experimental data available to quantify the reduction of hepatic and renal blood flows in paediatric CHF patients and to correlate them with the paediatric CHF classification system, the proposed changes in the paediatric CHF model were adopted from the previously mentioned adult values [9]. The relative reductions in the organ blood flows and their associations with the severity of CHF according to the NYHA classification and the Ross score are shown in Fig. 2.

\subsection{Pharmacokinetic/Clinical Data}

\subsubsection{In Adults}

The Medline database was screened for pharmacokinetic studies of carvedilol in healthy adults and CHF patients with known age, sex, height or weight data, clear dosing information and reported systemic drug concentration-time profiles. As a result, pharmacokinetic data from four different clinical studies in healthy subjects (3 studies totalling 41 subjects) and CHF patients (one study of ten patients with NYHA class III and ten patients with class IV CHF) were used in the adult model development and evaluation [13, 15-17]. These studies provided a total of nine data sets (five data sets in healthy subjects and four data sets in CHF patients) (Table 2). Each experimental data set represented a mean or median observed concentration-time profile in an average of 18 subjects who received either intravenous or oral doses of carvedilol. The average number of observed concentrations in these data sets was 13 (the total number was 122). These data sets were either provided by the author [15] or scanned from the publications' figures [13, 16, 17], using the digitizer tool in the data analysis and graphing software OriginPro ${ }^{\circledR}$ version 9.0 (OriginLab, Northampton, MA, USA). In addition, one study that reported only the pharmacokinetic parameters, but not the concentration-time profiles, after administration of oral suspension in healthy subjects was additionally used in the comparison of the observed and predicted values of the chosen pharmacokinetic parameters [18].

\subsubsection{In Children}

Two clinical data sets, including 32 paediatric CHF patients of different age groups with known age, sex, height and weight data, dosing information, Ross scores and measured plasma profiles were used (Table 3) [15]. The ages of the patients ranged from 43 days to 19.3 years (average 7.3 years), and they received either a $0.09 \mathrm{mg} / \mathrm{kg}$ single dose or $0.35 \mathrm{mg} / \mathrm{kg}$ steady-state doses of oral carvedilol for the treatment of CHF. The average number of measured concentrations in each patient was 12 (the total numbers were 197 and 202 for single-dose and steady-state sampling). In our paper, no explicit paediatric age groups were used, as the total number of the included paediatric patients was relatively small, with an unequal distribution between the different paediatric age subcategories. Whenever the term "children" is used, this refers to the entire paediatric age group ranging from birth to 18 years. The 19.3-year-old patient has been shown separately in the results, as this patient was out of the paediatric age range according to the guidelines set by the World Health Organization (WHO).

\subsection{Model Evaluation}

The simulations were performed by creation of a virtual population of 100 subjects for each clinical data set with the same age range, proportion of females, fluid intake and fasting/fed states as those in the reference clinical studies. The predictions performed with greater numbers of virtual 
Fig. 2 Changes in hepatic and renal blood flows in patients with heart failure, as incorporated into the developed physiologically based pharmacokinetic heart failure models of carvedilol. The presented data are taken from Leithe et al. [9]. NYHA New York Heart Association class

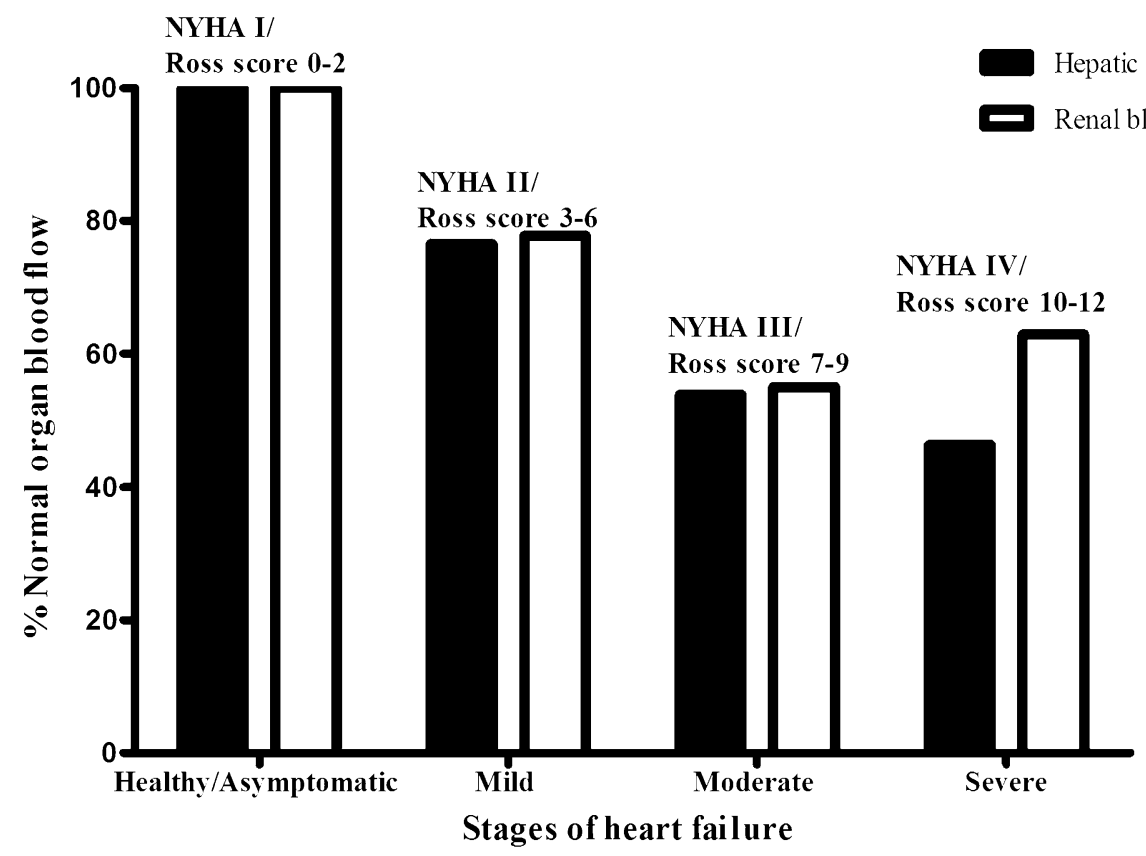

Table 2 Characteristics of the adult data sets used for carvedilol mode development

\begin{tabular}{|c|c|c|c|c|c|c|c|c|c|c|}
\hline \multirow[t]{2}{*}{ No. } & \multirow[t]{2}{*}{ Population } & \multirow{2}{*}{$\begin{array}{l}\text { Number of } \\
\text { subjects }\end{array}$} & \multirow{2}{*}{$\begin{array}{l}\text { Dose } \\
(\mathrm{mg})\end{array}$} & \multirow[t]{2}{*}{ Application } & \multicolumn{2}{|c|}{ Age (years) } & \multirow{2}{*}{$\begin{array}{l}\text { Proportion of } \\
\text { females }\end{array}$} & \multicolumn{2}{|c|}{ Body weight (kg) } & \multirow[t]{2}{*}{ References } \\
\hline & & & & & Mean & Range & & Mean & Range & \\
\hline 1 & Healthy & 12 & 5 & Intravenous infusion ${ }^{\mathrm{a}, \mathrm{b}}$ & - & $21-29$ & 0.25 & - & $59-92$ & [17] \\
\hline 2 & Healthy & 20 & 12.5 & Intravenous infusion $^{a}$ & - & $19-45$ & 0 & - & $60-92$ & {$[13]$} \\
\hline 3 & Healthy & 9 & $6.3^{\mathrm{c}}$ & Oral & $29.6^{\mathrm{d}}$ & $24-37$ & 0.66 & $69.8^{\mathrm{e}}$ & $56-100$ & {$[15]$} \\
\hline 4 & Healthy & 20 & 25 & Oral & - & $19-45$ & 0 & - & $60-92$ & {$[13]$} \\
\hline 5 & Healthy & 20 & 50 & Oral & - & $19-45$ & 0 & - & $60-92$ & {$[13]$} \\
\hline 6 & Healthy & 20 & 50 & $\mathrm{Oral}^{\mathrm{f}}$ & - & $19-45$ & 0 & - & $60-92$ & {$[18]$} \\
\hline 7 & Heart failure $\mathrm{g}, \mathrm{h}$ & 20 & 6.25 & Oral & $55^{\mathrm{i}}$ & $39-64^{i}$ & 0 & $89.5^{\mathrm{i}}$ & $60.8-113.1^{\mathrm{i}}$ & [16] \\
\hline 8 & Heart failure $\mathrm{g}, \mathrm{h}$ & 20 & 12.5 & Oral & $55^{\mathrm{i}}$ & $39-64^{i}$ & 0 & $89.5^{\mathrm{i}}$ & $60.8-113.1^{\mathrm{i}}$ & [16] \\
\hline 9 & Heart failure ${ }^{\mathrm{g}, \mathrm{h}}$ & 20 & 25 & Oral & $55^{\mathrm{i}}$ & $39-64^{i}$ & 0 & $89.5^{\mathrm{i}}$ & $60.8-113.1^{\mathrm{i}}$ & [16] \\
\hline 10 & Heart failure $\mathrm{g}^{\mathrm{g}, \mathrm{h}}$ & 20 & 50 & Oral & $55^{\mathrm{i}}$ & $39-64^{\mathrm{i}}$ & 0 & $89.5^{\mathrm{i}}$ & $60.8-113.1^{\mathrm{i}}$ & [16] \\
\hline
\end{tabular}

NYHA New York Heart Association

${ }^{a}$ The intravenous infusion was given over $1 \mathrm{~h}$

b The mean of the reported individual profiles was used for comparison

${ }^{\mathrm{c}}$ Dose administered as $0.09 \mathrm{mg} / \mathrm{kg}$ but normalized to total dose by multiplication by the average weight of the participants in the clinical trial

${ }^{\mathrm{d}}$ Median age 27 years, standard deviation 5.4, 25th and 75th percentiles 24.5 and 35 years

e Median weight $65 \mathrm{~kg}$, standard deviation 15.04, 25th and 75th percentiles 58.05 and $80.25 \mathrm{~kg}$

f Suspension

$\mathrm{g}$ The mean results reported in the study were further subdivided into NYHA class III and class IV subgroups

${ }^{\mathrm{h}}$ The population included 10 patients with NYHA class III and 10 with class IV heart failure

${ }^{\mathrm{i}}$ The presented values for age and weight are the reported values for the initial study population $(n=22)$

individuals (500 or 1000) produced no significant differences in comparison with the previous results (100 virtual individuals); therefore, as reported in other model-based PBPK studies [41-43], a virtual population of 100 individuals was always used for comparison with the observed profiles. Patient/population-specific genotype data were available from two clinical studies [15, 17], and the simulated virtual populations with use of model 2 had the same frequencies of genotypes as those in the reported clinical studies. 
Table 3 Characteristics of the paediatric data used for model development

\begin{tabular}{|c|c|c|c|c|c|c|}
\hline No. & Age (years) & Sex & Body weight (kg) & Single dose $(\mathrm{mg} / \mathrm{kg})$ & Steady-state dose $(\mathrm{mg} / \mathrm{kg})$ & Ross score/NYHA class \\
\hline \multicolumn{7}{|c|}{ Patients categorized according to their Ross score } \\
\hline 1 & 0.12 & Female & 3.1 & 0.09 & 0.35 & Score 3 \\
\hline 2 & 0.15 & Male & 3.9 & 0.09 & 0.35 & Score 3 \\
\hline $3^{\mathrm{a}}$ & 0.25 & Female & 4.2 & - & 0.35 & Score 4 \\
\hline 4 & 0.5 & Female & 5.2 & 0.09 & 0.35 & Score 8 \\
\hline $5^{\mathrm{b}}$ & 0.75 & Male & 8 & 0.09 & - & Score 3 \\
\hline 6 & 1.25 & Male & 10.1 & 0.09 & 0.35 & Score 3 \\
\hline 7 & 1.5 & Male & 9.5 & 0.09 & 0.35 & Score 10 \\
\hline 8 & 3.5 & Female & 13.1 & 0.09 & 0.35 & Score 3 \\
\hline $9^{\mathrm{b}}$ & 5.5 & Male & 20.2 & 0.09 & - & Score 3 \\
\hline 10 & 7.5 & Male & 24.3 & 0.09 & 0.35 & Score 5 \\
\hline 11 & 8.25 & Male & 25.8 & 0.09 & 0.35 & Score 7 \\
\hline 12 & 10.75 & Male & 25.5 & 0.09 & 0.35 & Score 4 \\
\hline 13 & 11.6 & Female & 34.3 & 0.09 & 0.35 & Score 4 \\
\hline 14 & 11.8 & Male & 39 & 0.09 & 0.35 & Score 2 \\
\hline $15^{\mathrm{a}}$ & 13.5 & Male & 52 & - & 0.35 & Not available \\
\hline \multicolumn{7}{|c|}{ Patients categorized according to their NYHA classification } \\
\hline 16 & 17.5 & Male & 56 & 0.09 & 0.35 & Class II \\
\hline 17 & 17.8 & Male & 61 & 0.09 & 0.35 & Class III \\
\hline 18 & $19.3^{\mathrm{c}}$ & Male & 98.2 & 0.09 & 0.35 & Class III \\
\hline Mean & 7.3 & & 27.41 & - & - & - \\
\hline Standard deviation & 6.76 & & 25.65 & - & - & - \\
\hline Median & 6.5 & & 22.25 & - & - & - \\
\hline 25 th percentile & 0.68 & & 7.3 & - & - & - \\
\hline 75th percentile & 12.23 & & 42.25 & - & - & - \\
\hline
\end{tabular}

All of the children were diagnosed with heart failure and were participants in the same clinical trial [15]

NYHA New York Heart Association

${ }^{a}$ Measured concentrations available only at steady state

b Measured concentrations available only after a single dose

${ }^{c}$ Patient out of the paediatric age range according to guidelines set by the World Health Organization

The evaluation of the developed PBPK models was performed by visual predictive checks and a comparison of the observed and predicted values of various pharmacokinetic parameters. In the visual predictive checks, the complete observed systemic drug concentration-time profile was overlaid on the predicted values so that a direct visual comparison could be made.

For the comparison of pharmacokinetic parameters, a non-compartmental analysis (NCA) was performed for each observed profile and its corresponding predicted value in each model (i.e. using the predicted values at the same time points), using Phoenix WinNonLin ${ }^{\circledR}$ version 6.3 software. The area under the systemic drug concentrationtime curve from time zero to the time of the last measured concentration $\left(\mathrm{AUC}_{\text {last }}\right)$ was calculated via the linear trapezoidal rule. The maximum concentration in a profile was defined as $C_{\max }$, and the clearance (CL for intravenous application and CL/F for oral application) was calculated by division of the given dose by the calculated $\mathrm{AUC}_{\text {last }}$.

Following the NCA, the observed/predicted ratio (ra-

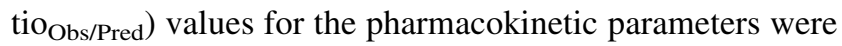
then calculated, and the final results were reported as mean ratio $_{(\mathrm{Obs} / \mathrm{Pred})}$ values with $95 \%$ confidence intervals (95\% CIs).

To the best of our knowledge, there are no clear guidelines regarding the error range that should be used during the evaluation of predictions obtained by PBPK models. The range most commonly used by researchers in this field is a 2-fold error range [3, 20, 44-47]. Others have also used a wider (3-fold) range [48] or a stricter (1.5-fold) range [49]. In our case, and because the models were used to perform predictions in both adults and children, and 
because of the high variability observed in the reported pharmacokinetic parameters of carvedilol, a 2-fold error range was used and was considered to be adequate.

Moreover, goodness-of-fit plots, population-predicted versus population-observed plots, residuals versus population-predicted plots and residuals versus time plots were used for identifying systemic errors in the developed models.

Depending upon the reported values of the pharmacokinetic parameters, the predicted mean or median values of the pharmacokinetic parameters were used for comparison and calculation of $\mathrm{ratio}_{(\mathrm{Obs} / \mathrm{Pred})}$ values.

\section{Results}

\subsection{Healthy Adults}

The observed and predicted systemic drug concentrationtime profiles after the administration of intravenous and oral carvedilol in healthy adults are shown in Fig. 3, and the residual plots are shown in Fig. 4. The results from both models were in good agreement with the observed data irrespective of the administered doses of 5-12.5 mg of intravenous carvedilol and $6.3-50 \mathrm{mg}$ of oral carvedilol. However, model 2 was superior to model 1 in terms of capturing the absorption phase, as model 1 tended to slightly overpredict early drug concentrations. This was further confirmed during comparison of the pharmacokinetic parameters, as the resultant mean $\mathrm{AUC}_{\text {last }}$ ratio(Obs/ Pred) values after intravenous application were 1.12 (95\% CI 1.01-1.22) and 0.97 (95\% CI 0.78-1.16), using models 1 and 2, respectively, and after oral application they were 0.80 (95\% CI $0.51-1.11)$ and 0.94 (95\% CI $0.65-1.23$ ), using models 1 and 2 , respectively. In addition, the calculated mean ratio ${ }_{(\mathrm{Ob} / \mathrm{Pred})}$ values for $\mathrm{AUC}_{\text {last }}, C_{\mathrm{max}}$, $\mathrm{CL}$ and $\mathrm{CL} / F$ were within the defined 2-fold error range (Fig. 5a-c).

\subsection{Adult Heart Failure Patients}

The observed and predicted systemic drug concentrationtime profiles after administration of steady-state oral doses of carvedilol $(6.25-50 \mathrm{mg})$ in NYHA class III and class IV CHF patients are shown in Fig. 3. The visual predictive checks show a substantial improvement in the predictions after incorporation of reduced organ blood flows in the models. The mean $\mathrm{AUC}_{\text {last }} \mathrm{ratio}_{(\mathrm{Obs} / \mathrm{Pred})}$ values without adjustment of the organ blood flows were outside the 2-fold error range in both models, i.e. 2.33 (95\% CI 2.02-2.63) in model 1 and 2.65 (95 \% CI 2.29-3.01) in model 2. After incorporation of the proposed organ blood flow changes, these ratios improved to 0.79 (95\% CI 0.69-0.90) and
Fig. 3 Comparison of observed and predicted systemic carvedilol concentration-time profiles in healthy subjects and heart failure patients after intravenous (iv) or oral drug dosing. Healthy adults, iv application: a, b $5 \mathrm{mg}$ [17], c, d $12.5 \mathrm{mg}$ [13]. Healthy adults, oral application: e, f $0.09 \mathrm{mg} / \mathrm{kg}$ [15], g, h $25 \mathrm{mg}$ [13], i, j $50 \mathrm{mg}$ [13]. Heart failure patients, oral application: k, l $6.25 \mathrm{mg}$ [16], m, n $12.5 \mathrm{mg}, \mathbf{o}, \mathbf{p} 25 \mathrm{mg}, \mathbf{q}, \mathbf{r} 50 \mathrm{mg}$. The observed data are shown as mean values $(\mathbf{a}, \mathbf{b}, \mathbf{k}-\mathbf{r})$ or as median values $(\mathbf{c}-\mathbf{j})$ of the study population. Standard deviations were available only for $\mathbf{e}, \mathbf{f}$. Prediction results are shown for model 1 or model 2 as median or mean values (solid lines), 5th and 95th percentiles (dotted lines) and minimum/maximum values (dashed lines). Prediction results in heart failure patients $(\mathbf{k}-\mathbf{r})$ are for the modified model with reduced organ blood flow, whereas dashed grey lines indicate mean model predictions without incorporation of organ blood flow changes

1.03 (95\% CI 0.89-1.16), respectively. The complete comparison of the calculated pharmacokinetic parameters with and without adjustment of organ blood flows is shown in Fig. 5a-c. Similar results were seen after further categorization of the CHF patients into NYHA class III and class IV subgroups. The developed models were capable of predicting carvedilol exposure in NYHA class III and class IV patients, as can be seen by the mean $\mathrm{ratio}_{(\mathrm{Ob} / \mathrm{Pred})}$ values and their $95 \%$ CIs (Fig. 5d-f). Despite the fact that the predicted concentrations in both models were within a 2-fold error range (Fig. 4), it can be noticed that model 1 tended to overpredict drug concentrations in the absorption phase at all dosage levels, whereas model 2 predictions were in closer agreement with the observed data.

Similarly, the mean CL/F $\mathrm{ratio}_{(\mathrm{Obs} / \text { Pred) }}$ values without reduction of organ blood flows were outside the 2-fold error range, i.e. 0.34 (95\% CI 0.31-0.37), in both models, and were significantly improved to 1.0 (95\% CI $0.92-1.08)$ and 0.95 (95\% CI 0.88-1.03) in models 1 and 2 , respectively. The predicted bioavailability $(F)$ and $F_{\mathrm{h}}$ decreased with a decrease in $Q_{\mathrm{H}}$ (increase in severity of CHF). The predicted effect of the decreased $Q_{\mathrm{H}}$ on carvedilol $\mathrm{CL} / F$ and bioavailability in the different stages of heart failure can be seen in Fig. S1 in the Electronic Supplementary Material.

\subsection{Paediatric Heart Failure Patients}

Carvedilol exposure after single and steady-state doses of 0.09 and $0.35 \mathrm{mg} / \mathrm{kg}$, respectively, was predicted using both models in the entire paediatric age range (see Fig. S2 in the Electronic Supplementary Material). It can be seen that both models were able to describe the individual plasma concentration-time points; however, model 2 was again better when compared with model 1 , with a greater number of observed plasma concentration-time points within the maximum and minimum prediction range ( $\sim 93 \%$ for model 2 versus $\sim 86 \%$ for model 1 ). 

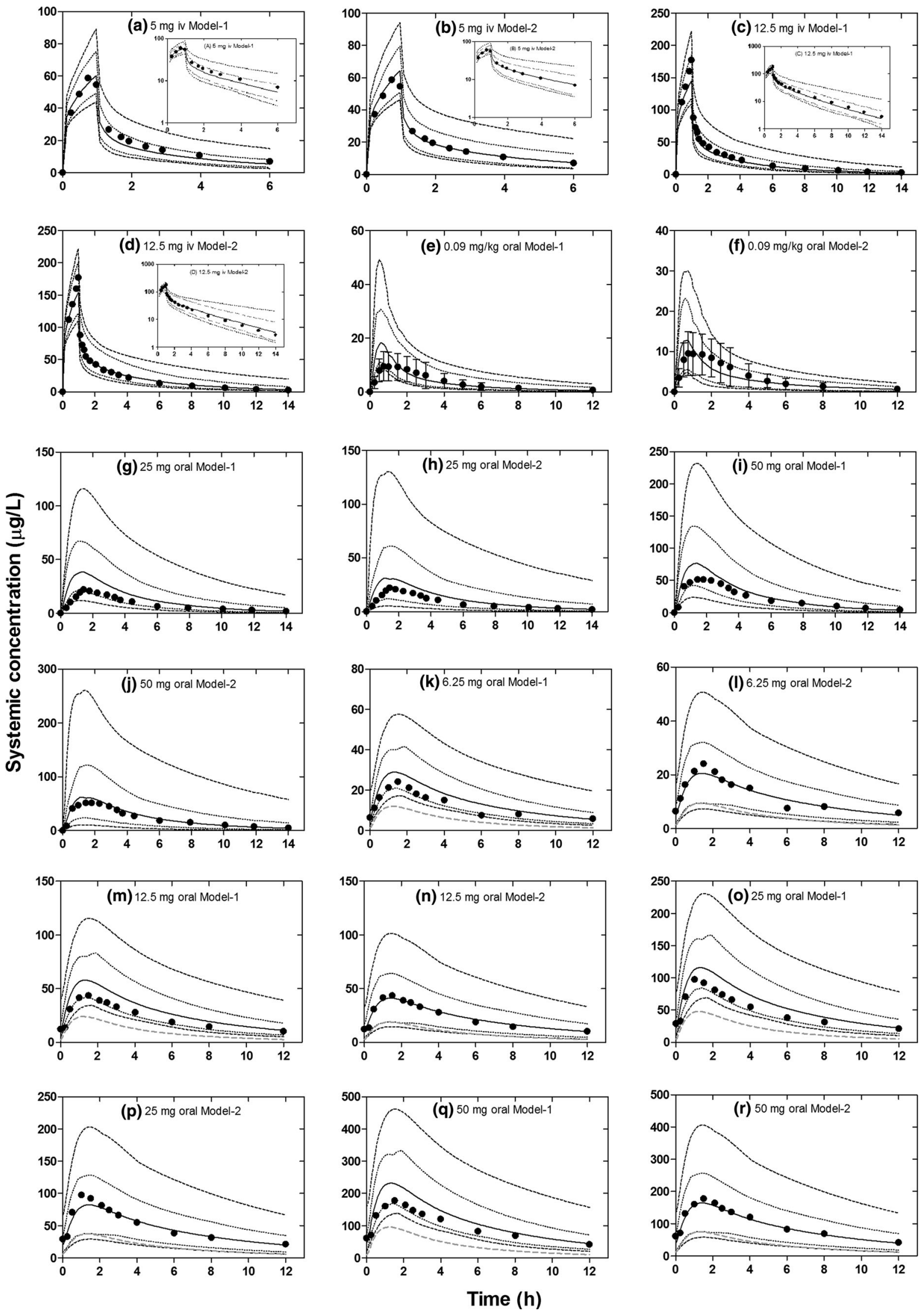


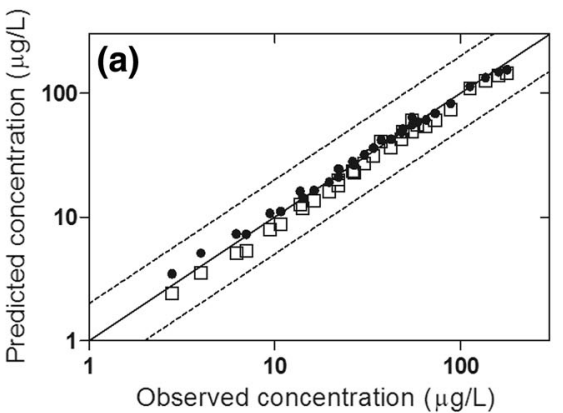

iv dosing in healthy adults
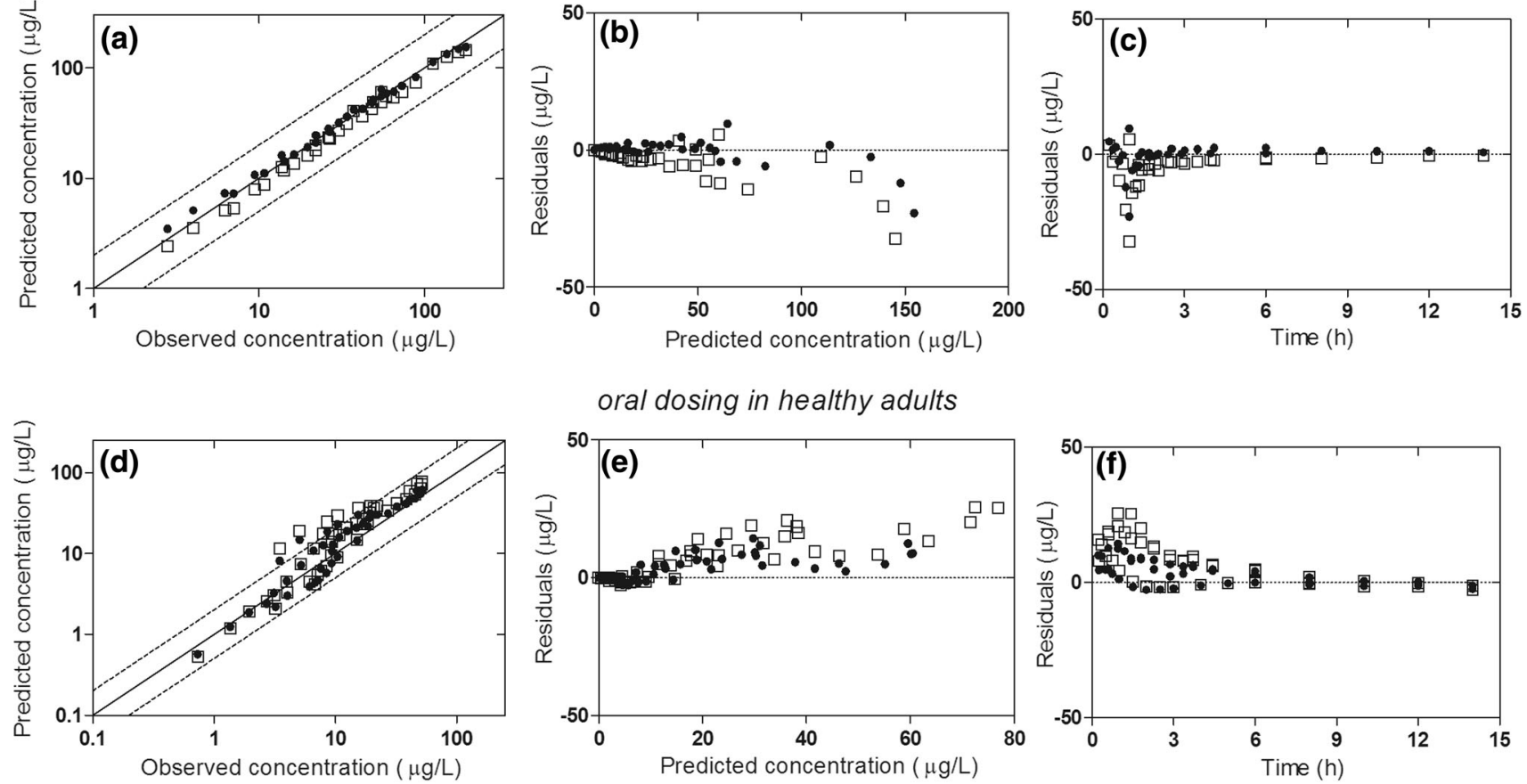

oral dosing in healthy adults
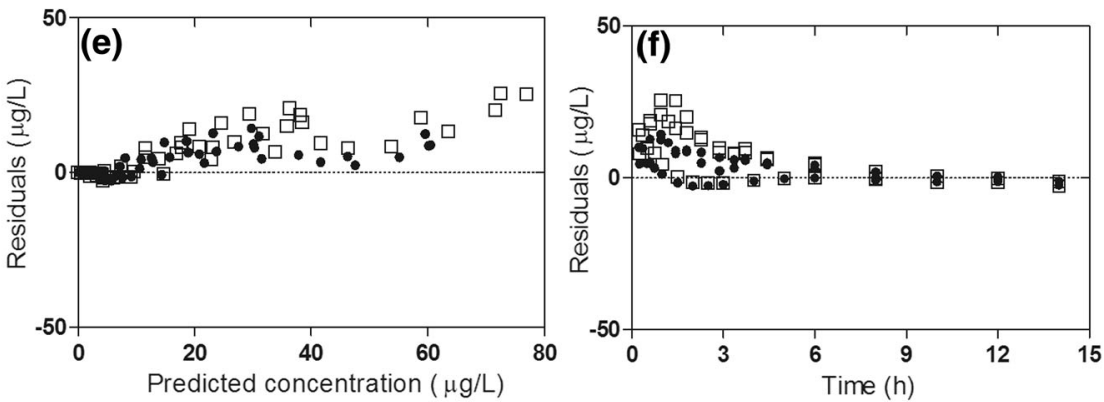

oral dosing in heart failure without organ blood flow reductions
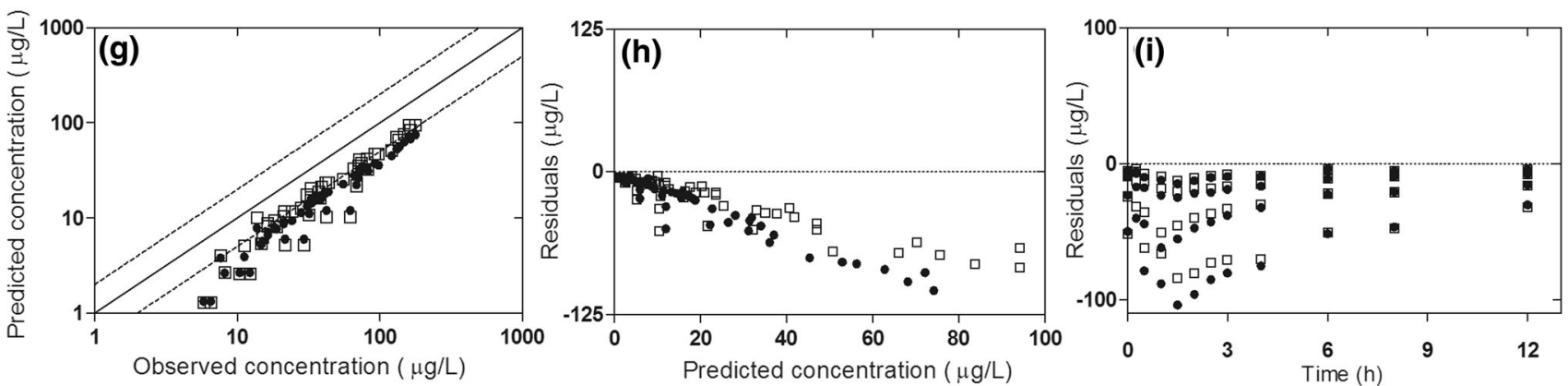

oral dosing in heart failure with organ blood flow reductions
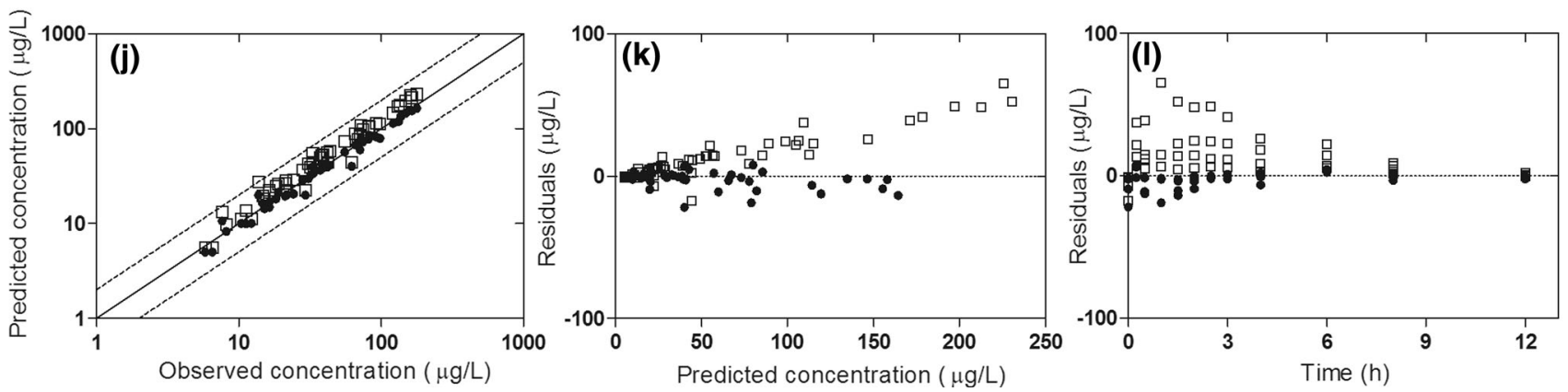

Fig. 4 Goodness-of-fit plots for model predictions in the adult population, presented as $\mathbf{a}, \mathbf{d}, \mathbf{g}, \mathbf{j}$ predicted versus observed concentration plots; $\mathbf{b}, \mathbf{e}, \mathbf{h}, \mathbf{k}$ residuals versus predicted concentration plots; and c, f, i, l residuals versus time plots. a-c Intravenous (iv) dosing in healthy adults. d-f Oral dosing in healthy adults. $\mathbf{g}-\mathbf{i}$ Oral dosing in heart failure patients without renal and hepatic blood flow reductions. $\mathbf{j}-\mathbf{l}$ Oral dosing in heart failure patients with renal and hepatic blood flow reductions. Solid lines indicate lines of identity, dashed lines indicate 2 -fold error ranges, open squares indicate results from model 1 and filled circles indicate results from model 2 
(a)

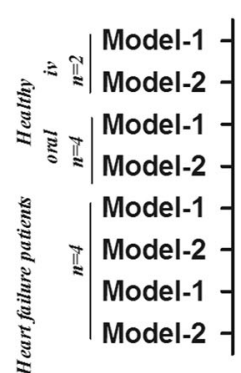

$\mathrm{AUC}_{\text {last }}$

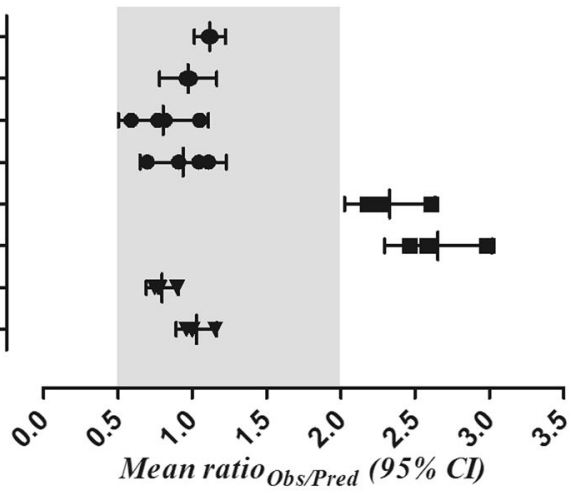

(b)

$\mathrm{C}_{\max }$
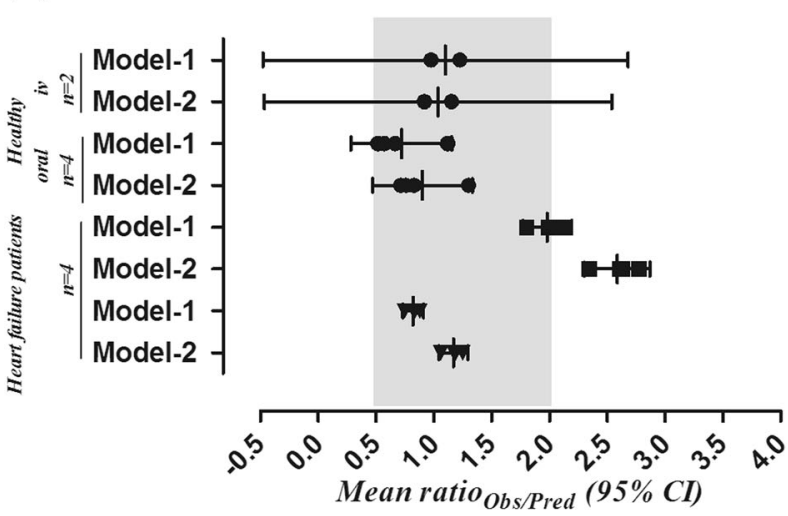

(c)

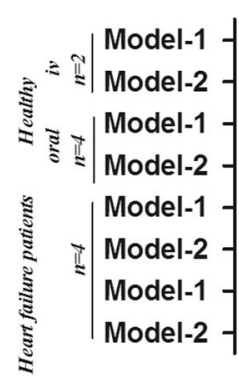

Clearance

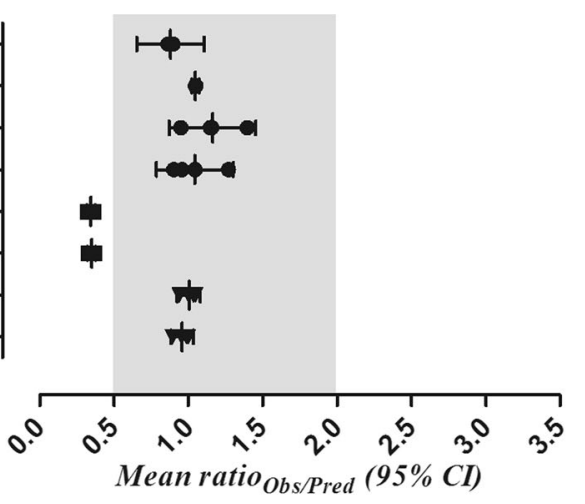

Fig. 5 Comparison of observed and predicted values of the area under the systemic drug concentration-time curve from time zero to the time of the last measured concentration $\left(\mathrm{AUC}_{\text {last }}\right)$, the maximum concentration $\left(C_{\max }\right)$ and calculated oral drug clearance $(\mathrm{CL} / F)$ in healthy adult and heart failure populations. Results are presented as individual and mean observed/predicted ratio [ $\left.\mathrm{ratio}_{(\mathrm{Obs} / \mathrm{Pred})}\right]$ values with $95 \%$ confidence intervals (95\% CIs), using model 1 and model 2. a-c Filled circles indicate healthy adults, filled squares indicate heart failure patients without renal and hepatic blood flow

The individual predictions made by model 2 after administration of a single oral dose of carvedilol $0.09 \mathrm{mg} / \mathrm{kg}$ to 15 paediatric patients and one young adult are shown for (d)

$\mathrm{AUC}_{\text {last }}$
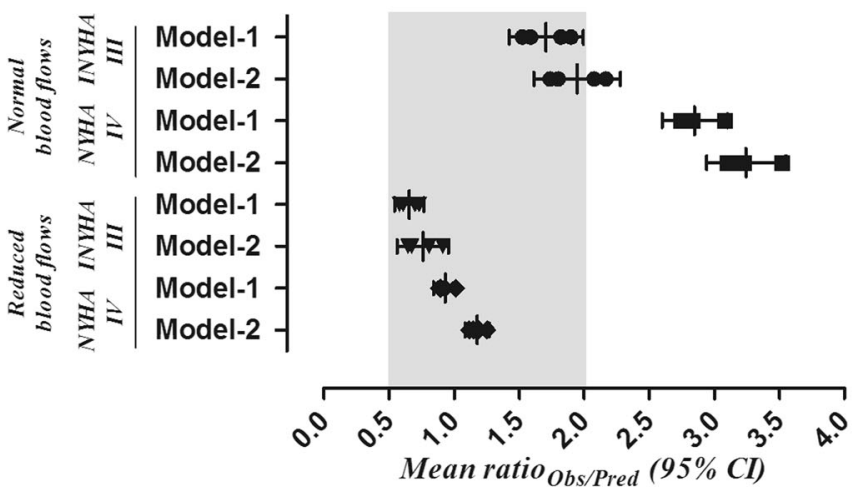

(e)

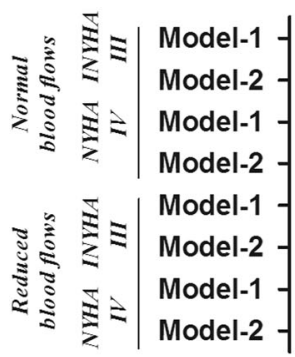

$\mathrm{C}_{\max }$

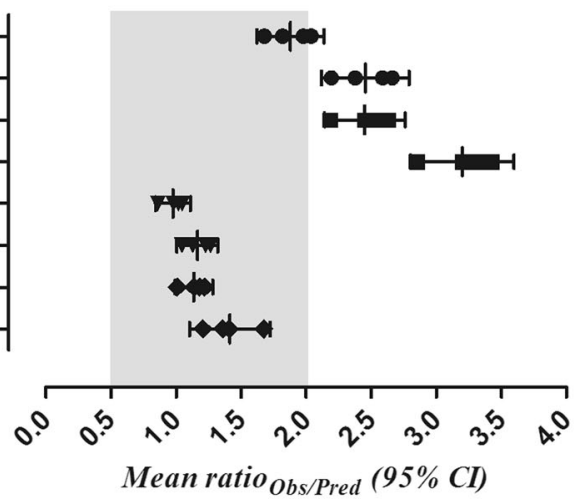

(f)

Clearance
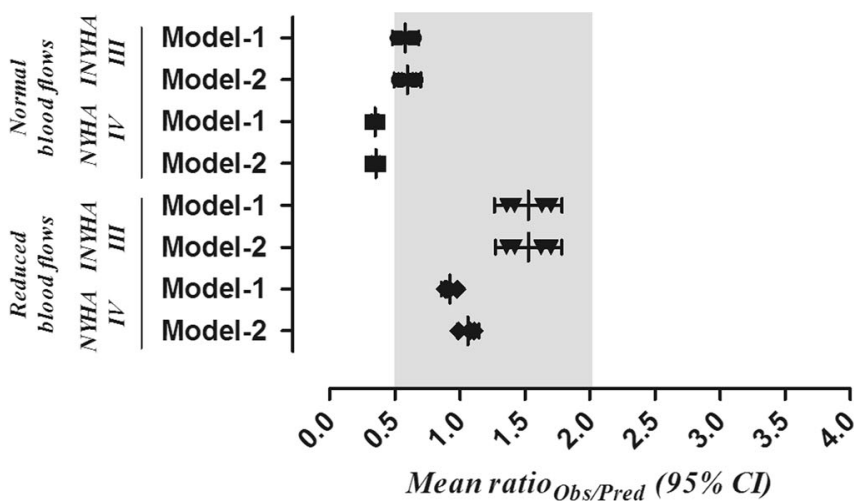

reductions, and inverted filled triangles indicate heart failure patients with renal and hepatic blood flow reductions. d-f Filled circles and filled squares indicate heart failure patients in New York Heart Association (NYHA) class III and class IV, respectively, without renal and hepatic blood flow reductions; inverted filled triangles and diamonds indicate heart failure patients in NYHA class III and class IV, respectively, with renal and hepatic blood flow reductions. The grey shaded areas indicate 2-fold error ranges. $i v$ intravenous, $n$ number of data sets

a visual predictive check in Fig. 6, with and without reduction of organ blood flows. In addition, the residual plots are given in Fig. 7. In general, the model was able to 

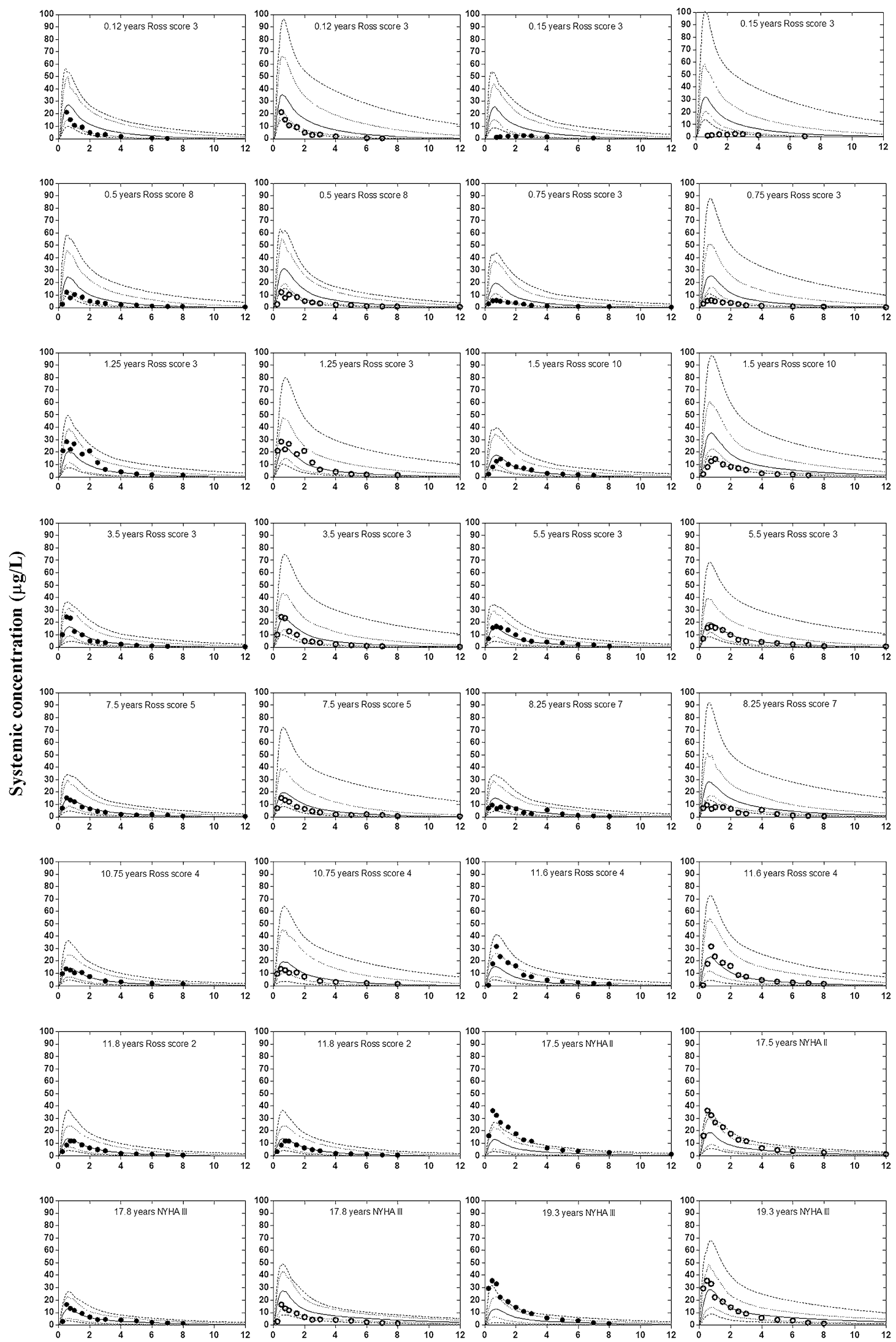

Time(h) 
4Fig. 6 Individual predictions made by model 2 after administration of a single oral test dose of carvedilol $0.09 \mathrm{mg} / \mathrm{kg}$ with adjustment of organ blood flows (shown by open circles) and without adjustment of organ blood flows (shown by filled circles). Solid lines indicate median predictions, dashed lines indicate minimum and maximum predictions, dotted lines indicate 5th and 95th percentiles, and filled and open circles indicate observed data [15]. NYHA New York Heart Association

predict carvedilol concentrations accurately in all patients above 1 year of age (12 out of 16). For the one patient with symptomatic CHF (a Ross score of 0-2), no changes in blood flows were incorporated; therefore, there was no change to be seen in the visual predictive check. For the ten paediatric patients with mild $\mathrm{CHF}$, nine were with Ross scores of 3-6, the incorporation of blood flow reductions did not improve the predictions in these patients but improvement was seen in one patient classified with NYHA class II (see also the pharmacokinetic comparison). Concerning the four patients with moderate $\mathrm{CHF}$, the model predictions were better without organ blood flow reductions in those patients classified with Ross scores ( $n=2$, with scores of 7-9), whereas in the remaining two who were classified as being in NYHA class III, the prediction was clearly improved in one patient (19.3-year-old patient) but no improvement was seen in other NYHA III patient (17.8-year-old patient) with reductions in organ blood flows.

The mean $\mathrm{AUC}_{\text {last }}$ ratio $(\mathrm{Obs} / \mathrm{Pred})$ values and $95 \% \mathrm{CIs}$ after administration of a single dose $(0.09 \mathrm{mg} / \mathrm{kg})$ of carvedilol were 0.98 (95\% CI 0.68-1.28) and 1.0 (95\% CI 0.7-1.30) without incorporation of reduced organ blood flows. There was no improvement seen in the prediction capability of both models with reduction of organ blood flows, after administration of single and steady-state doses of carvedilol, as the calculated $\mathrm{ratio}_{(\mathrm{Obs} / \mathrm{Pred})}$ values for $\mathrm{AUC}_{\text {last }}, C_{\max }$ and $\mathrm{CL} / F$ were further away from unity (Fig. 8). The previous finding that predictions in adult $\mathrm{CHF}$ patients, classified with NYHA system improved after reduction of organ blood flows was also seen here in two patients (17.5 years NYHA II and 19.3 years NYHA III).
Fig. 7 Goodness-of-fit plots for model 2 predictions in the paediatric population, presented as a, $\mathbf{d}$ median predicted versus individual observed concentrations plots; $\mathbf{b}$, e residuals versus median predicted concentrations plots; and $\mathbf{c}, \mathbf{f}$ residuals versus time plots. a-c Results without renal and hepatic blood flow reductions, shown by filled circles. d-f Results with renal and hepatic blood flow reductions, shown by open circles. Solid lines indicate lines of identity, and dashed lines indicate 2 -fold error ranges
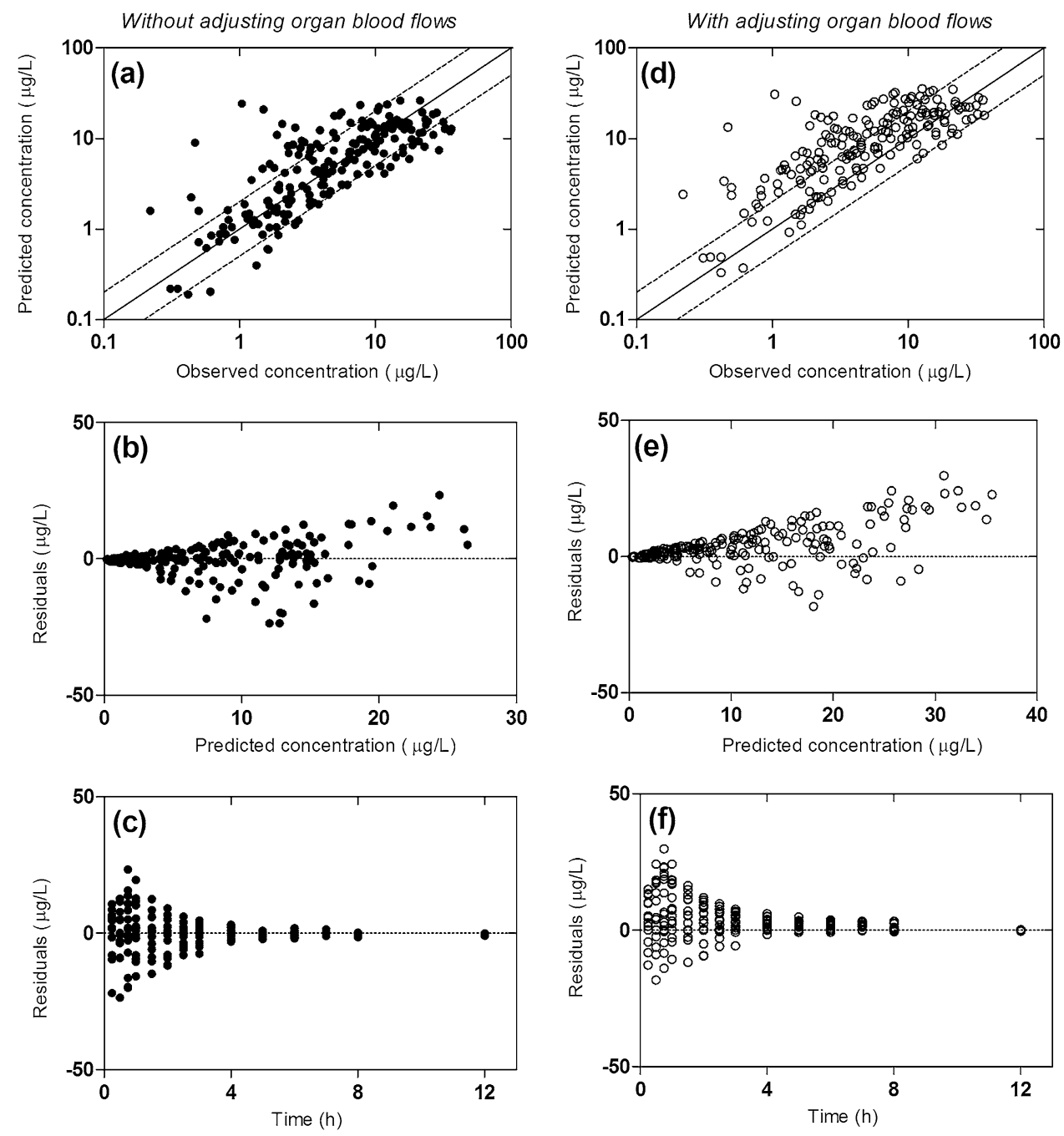
Stratified by age

(a)

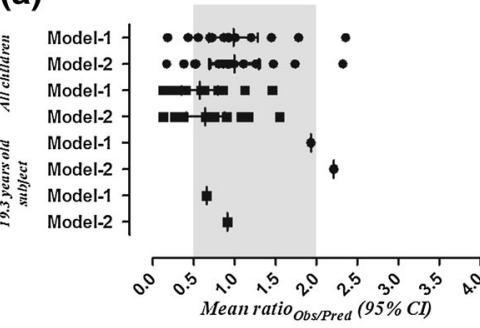

(b)

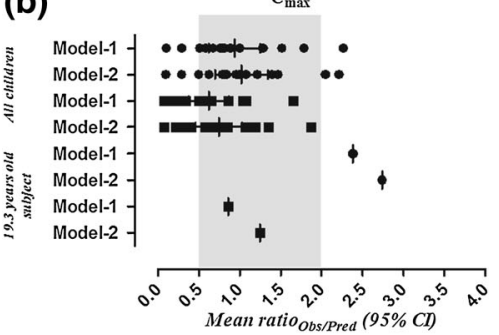

(c)

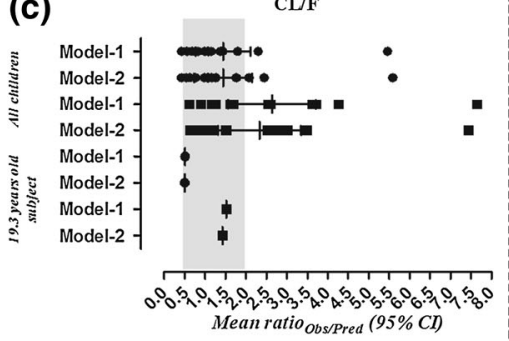

Stratified by classification of the disease severity
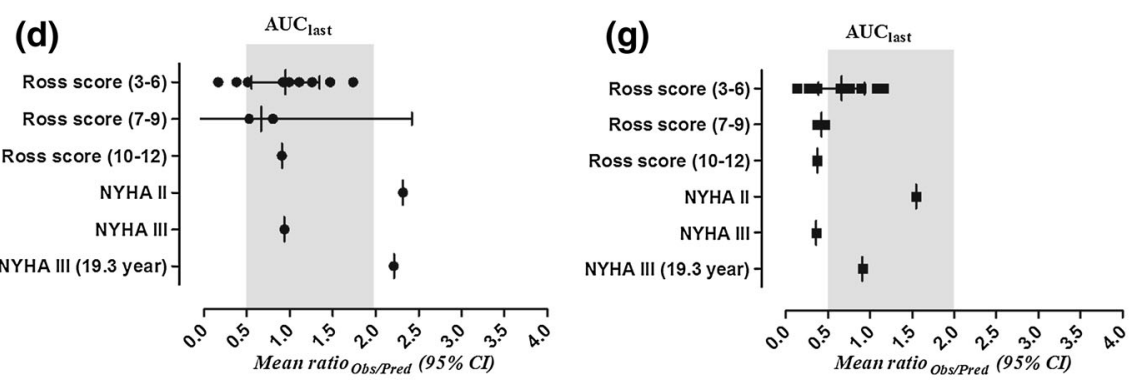

(e)

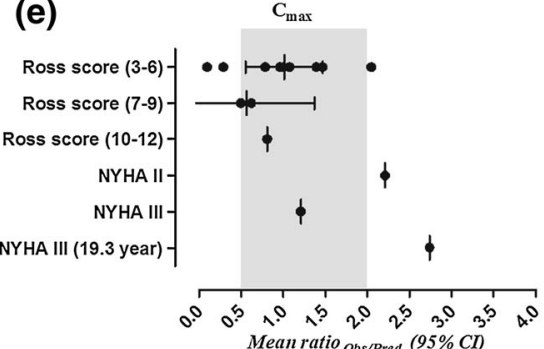

(h)
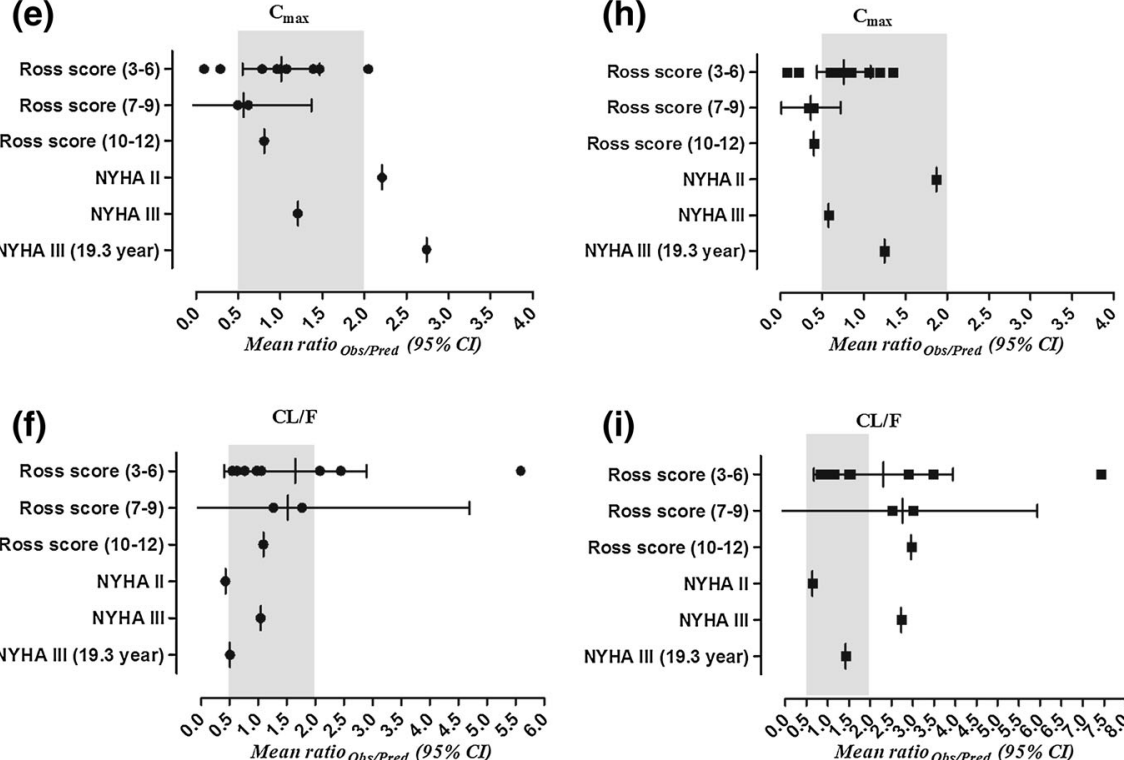

(i)

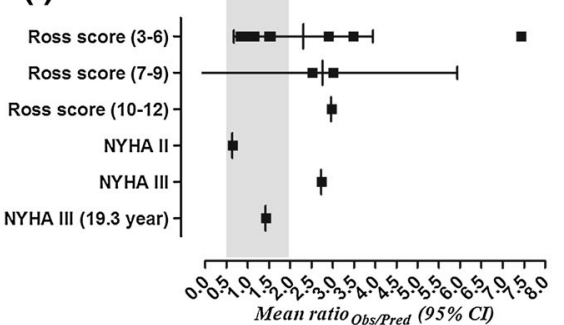

Fig. 8 Comparison of observed and predicted values of the area under the systemic drug concentration-time curve from time zero to the time of the last measured concentration $\left(\mathrm{AUC}_{\text {last }}\right)$, the maximum concentration $\left(C_{\max }\right)$ and oral drug clearance $(\mathrm{CL} / F)$ in paediatric heart failure patients after administration of a single dose of $0.09 \mathrm{mg} /$ $\mathrm{kg}$. The results are presented as individual and mean observed/ predicted ratio $\left[\mathrm{ratio}_{(\mathrm{Obs} / \mathrm{Pred})}\right]$ values with $95 \%$ confidence intervals

The observed carvedilol CL/ $F$ increased with age after administration of the single dose of $0.09 \mathrm{mg} / \mathrm{kg}$, starting from $9.20 \mathrm{~L} / \mathrm{h}$ in a patient aged 0.12 years and reaching up to $110 \mathrm{~L} / \mathrm{h}$ in a patient aged 19.3 years. When the $\mathrm{CL} /$ $F$ was normalized for weight, a decrease was seen with increasing age, as it decreased from $2.94 \mathrm{~L} / \mathrm{h} / \mathrm{kg}$ at the age of 0.12 years to $1.12 \mathrm{~L} / \mathrm{h} / \mathrm{kg}$ until the age of 19.3 years, which is a reflection of the incorporated knowledge of enzyme ontogeny in the modelling software. A similar trend was seen after administration of steady-state target doses of carvedilol. The developed models were capable of capturing the age-related changes in carvedilol CL/ $F$ after single and steady-state administration (see Fig. S3 in the Electronic Supplementary Material).
(CIs), without blood flow reductions (shown by filled circles) and with blood flow reductions (shown by filled squares). The grey shaded areas indicate 2-fold error ranges. a-c Results from both models, stratified by age. $\mathbf{d}-\mathbf{i}$ Results from model 2, stratified by the severity of the disease. NYHA New York Heart Association class

\section{Discussion}

In this study, reduced hepatic and renal blood flows were incorporated into whole-body PBPK models to predict carvedilol exposure in CHF patients. Both of the developed models were able to describe carvedilol pharmacokinetics in adults and paediatric subjects over 1 year of age, but model 2 was shown to be superior to model 1 in its predictive performance. The incorporation of the reduced hepatic and renal blood flows into the developed $\mathrm{CHF}$ models resulted in a significant improvement in predictions of drug exposure in adult patients. Conversely, the incorporated blood flow reductions did not result in any improvements in the paediatric CHF patients, except in those 
patients who were categorized according to NYHA classification of heart failure (NYHA II and NYHA III) as in adults.

Following the implemented strategy of model development, the presented models were first parameterized and evaluated in healthy adults. The results showed accurate prediction of carvedilol exposure over a wide range of administered intravenous and oral doses (Figs. 3, 4, 5), which supported the ability of the models to account for the various processes governing drug absorption and disposition. The predicted bioavailability in healthy volunteers was in the range of 15-27\% according to both models, which was quite close to the reported values of 22-24\% [18]. Furthermore, the mean $\mathrm{AUC}_{\text {last }}$ ratio $_{(\mathrm{Obs} / \mathrm{Pred})}$ values in healthy adults were within the 2-fold error range, i.e. after intravenous application: 1.12 (95\% CI 1.01-1.22) and 0.97 (95\% CI 0.78-1.16), using models 1 and 2, respectively; and after oral application: 0.80 (95\% CI 0.51-1.11) and 0.94 (95\% CI 0.65-1.23), respectively.

In CHF, hepatic and renal blood flows decrease with increasing severity of the disease [9]. Incorporation of these reductions improved the model predictions significantlyfor example, the mean $\mathrm{AUC}_{\text {last }}$ ratio $_{(\mathrm{Obs} / \mathrm{Pred})}$ values changed from 2.33 (95 \% CI 2.02-2.63) to 0.79 (95 \% CI 0.69-0.90) and from 2.65 (95\% CI 2.29-3.01) to 1.03 (95\% CI 0.89-1.16), using models 1 and 2, respectively. The extensive first-pass effect and the high hepatic extraction ratio of carvedilol $[11,13,18]$ imply that changes in $Q_{\mathrm{H}}$, as seen in $\mathrm{CHF}$, will influence its first-pass and systemic hepatic metabolism - and thus its bioavailability and total exposure. The reduced $Q_{\mathrm{H}}$ will lead to an increase in first-pass metabolism, with a subsequent decrease in drug bioavailability (because of higher hepatic extraction during the first pass) and a decrease in the systemic clearance of the drug. The reduction in carvedilol bioavailability and $\mathrm{CL} / F$ with the increased severity of CHF was clearly seen in the predictions (see Fig. S1 in the Electronic Supplementary Material). On the other hand, the $\mathrm{CL}_{\mathrm{R}}$ of carvedilol is only about 1-2\% $[13,34]$ of its total clearance; therefore, the change in the latter can clearly be attributed to the decrease in $\mathrm{CL}_{\mathrm{H}}$. The ratio $_{(\mathrm{Obs} / \mathrm{Pred})}$ values and $95 \%$ CIs for the pharmacokinetic parameters after further subdivision of the adult CHF patients into NYHA class III and class IV categories supported our hypothesis that the incorporated reduced hepatic and renal blood flows in the developed models are associated with the NYHA classification of CHF (Fig. 5).

The previously presented and evaluated models in adults formed the basis for extrapolation of carvedilol pharmacokinetics to children on a physiological basis, using the paediatric module in Simcyp ${ }^{\circledR}$. The developed paediatric models, which incorporate a wide range of age-specific information, were capable of reflecting carvedilol exposure after single and steady-state doses in the paediatric age range (see Fig. S2 in the Electronic Supplementary Material). However, it was noticed that the models tended to generally overpredict the drug concentrations in those patients under 1 year of age, with or without incorporation of blood flow reductions. An overprediction of drug concentrations in this age group was previously reported when child-specific PBPK models were used to predict the pharmacokinetics of drugs when they were given orally [20], which was mostly attributed to age-specific information gaps in the parameterization of the integrated absorption models. Second, in contrast to the adult patients, the improvement in the model predictions with incorporation of blood flow changes was limited to a few cases, as is discussed below.

Ten out of the 14 paediatric CHF patients who were included in the clinical trial were diagnosed with mild $\mathrm{CHF}$ — nine patients according to the modified Ross scoring system and one patient according to the NYHA classification. For patients categorized with Ross score, no substantial improvement in predictions were seen, on the other hand prediction was improved in one patient who was staged NYHA II when reduced organ blood flows were incorporated (Fig. 6). These findings could suggest that the pharmacokinetics of carvedilol in young children may not be affected in mild CHF as they are in moderate or severe CHF. On the other hand, no improvement was seen when the model was modified for blood flow changes in the only patient with severe CHF, as indicated by a Ross score of 10 , whereas clear improvement was only seen in 19.3 years patient staged as NYHA class III and the 17.8 years patient staged as NYHA class III was better described without organ blood flow reductions. As a result, an assumption that the Ross scoring system is poorly correlated with the reductions in organ blood flows in comparison with the NYHA classification for grading CHF cannot be completely excluded. In the modified Ross score system, different subjects with different symptoms may end up having the same score and CHF category. Among the six categories used for classification of paediatric patients according to the modified Ross scoring system, three are related to breathing, which may result in undermining of the other symptoms associated with $\mathrm{CHF}$ in paediatric patients [50]. In addition, the aetiology and the pathophysiology of CHF are different in adults when compared with children, especially in the first few years after birth. Children have higher heart rates [51] than adults [52], which decease with age after birth and become comparable to those of adults at the age of 18 years [51]; therefore, children may have a greater capacity to compensate for a decrease in cardiac output. Our paediatric disease model is based on the adult heart failure model with its incorporated reductions in organ blood flows, because in paediatrics, we are not aware of any study that has quantified hepatic and renal blood flow reductions with respect to different stages 
of heart failure in paediatric patients. Therefore, these adult quantifications may not be completely true for children. Finally, children also have a higher percentage of liver weight to total body weight when compared with adults [53], which may lead to a higher drug clearance capacity (by rapidly maturating and abundant metabolizing enzymes), and decreased $Q_{\mathrm{H}}$ may not have the same impact on drug clearance as it does in adults.

The observed weight-normalized carvedilol CL/F decreased in paediatric patients with increasing age (from 43 days to 17.8 years). This was due to the fact that infants have a liver weight of around $4 \%$ of their total body weight, which decreases to about $2 \%$ in adults [53]. Moreover, CYP2D6 is the major enzyme involved in carvedilol metabolism $(\sim 60 \%)$, and it achieves more than $50 \%$ of its adult activity within the first month of life [37]. Another enzyme that plays a minor role in carvedilol metabolism is CYP2C9, which also has a fast ontogeny; therefore, it was previously reported that significantly higher weight-normalized doses, as compared with those used in adults, are required in young children receiving drugs that are primarily metabolized by this enzyme [54]. Furthermore, the decrease in carvedilol CL/F can also be attributed to decreases in organ blood flows, as improvement in predictions were seen with incorporation of reduced organ blood flows in patients within the adolescent age group (Fig. 6). The developed paediatric models were capable of capturing the age-specific changes in carvedilol clearance, as the mean $\mathrm{CL} / F \mathrm{ratio}_{(\mathrm{Obs} / \mathrm{Pred})}$ values after administration of a single dose of carvedilol, without incorporation of reduced organ blood flows, were 1.44 (95\% CI 0.77-2.11) and 1.43 (95\% CI 0.72-2.14) with use of models 1 and 2, respectively (Fig. 8).

Despite the fact that the already incorporated ontogeny profiles for each of the individual CYP enzymes gave model 2 a clear advantage over model 1 through its ability to incorporate enzyme-specific genotype data, the predicted results obtained by both models were very similar. One reason could be that the customized ontogeny profile that was assigned in model 1 is based on the ontogeny data of each of the individual enzymes used in model 2. A second reason is that the additional superiority of model 2 could have been clearer if population-specific enzyme genotype data had been included for all of the clinical studies that were used. In our study, this was done in the simulations of just two clinical studies $[15,17]$.

\section{Limitations}

Some of the carvedilol concentration-time profiles observed in adults were scanned from the publications' figures rather than being obtained from their sources.
However, the difference seen between the reported pharmacokinetic parameters in the original papers and those calculated by us using the scanned profiles was negligible and therefore of no significance to the model evaluation results. In addition, the adult $\mathrm{CHF}$ model was evaluated only with NYHA class III and class IV patients, but this was because of the availability of only one pharmacokinetic study of carvedilol in CHF patients.

The absorption (ADAM) model used for paediatric simulations was not completely parameterized with agespecific information for all of the anatomical and physiological factors that may influence drug absorption. For example, the model contains information on age-related changes in intestinal length, diameter, blood flows and intestinal CYP enzymes but no information on changes in gastric and intestinal $\mathrm{pH}$, bile secretion, transporters and gut fluid dynamics. This reflects the fact that paediatric drug absorption in PBPK modelling software is an area of ongoing research; therefore, the presented paediatric results must be judged in this context. The fluid intake with the dose was not modified according to the age of the paediatric subjects; however, no relevant impact on the results from the four infants under 1 year of age was seen. The assigned $P_{\text {eff,man }}$ value was not changed with age, and so a similar passive diffusion of the substance was assumed in adults and children.

The role of the three different UGT enzymes involved in carvedilol metabolism was assigned collectively as an additional clearance. Since each of these pooled UGT enzymes possesses a different ontogeny profile, and we have no clear information about their individual contributions, no specific ontogeny was assigned to this collective value. This could be a limitation in model 2 and an aspect for improvement of the model in the future.

Finally, in the presented models, we assumed that organ blood flow reductions in paediatric heart failure patients were similar to those in adults, as no specific clinical information was available for the paediatric population.

\section{Conclusion}

PBPK models incorporating hepatic and renal blood flow changes were developed and evaluated for their ability to predict carvedilol exposure in both adults in children. The incorporation of reduced blood flows into the developed PBPK models significantly improved the prediction of pharmacokinetic parameters of carvedilol in adult $\mathrm{CHF}$ patients and established a clear relationship between organ blood flow reductions and the severity of $\mathrm{CHF}$ assessed by the NYHA classification of CHF. The predictions in paediatric patients with $\mathrm{CHF}$ were not improved with reductions in organ blood flows, and it was hypothesized that the 
paediatric system for assessing the severity of heart failure (the Ross score) was not as well correlated with the organ blood flow reductions occurring in the disease as the NYHA classification. Since the majority of the paediatric patients in the clinical trial were diagnosed with mild heart failure (with Ross scores of 3-6), it was assumed that the pathophysiology of the disease in young children is different in mild CHF; this assumption was further strengthened when the organ blood flow reductions in children above 17 years of age, assessed by the NYHA classification, resulted in improvements in the predicted pharmacokinetic parameters. However, these results cannot be generalized to all of the paediatric CHF patients, taking into consideration the small sample size of the paediatric clinical trial data used for model development and, secondly, in terms of the severity of disease, the participants were not evenly distributed throughout the paediatric age range. In order to draw a definite conclusion regarding the role of reduced organ blood flows in children, there is a need for further evaluation with additional clinical pharmacokinetic data in paediatric patients with different degrees of $\mathrm{CHF}$.

Because of the mechanistic nature of the presented PBPK models, they could be extended to other high-extraction drugs in heart failure patients, or they could serve as a basis for development of a PBPK model of carvedilol and similar high-extraction drugs in special populations, such as geriatric patients, patients with renal insufficiency or patients with liver failure.

Acknowledgments The research leading to these results has received funding from the European Union Seventh Framework Programme (FP7/2007-2013) under Grant agreement No. 602295 (LENA). The authors thank Certara for providing academic licenses for the Simcyp ${ }^{\circledR}$ and WinNonLin ${ }^{\circledR}$ software programs.

Open Access This article is distributed under the terms of the Creative Commons Attribution Noncommercial License which permits any noncommercial use, distribution, and reproduction in any medium, provided the original author(s) and the source are credited.

\section{References}

1. Boucher BA, Wood GC, Swanson JM. Pharmacokinetic changes in critical illness. Crit Care Clin. 2006;22(2):255-71, vi.

2. Johnson TN, Boussery K, Rowland-Yeo K, Tucker GT, RostamiHodjegan A. A semi-mechanistic model to predict the effects of liver cirrhosis on drug clearance. Clin Pharmacokinet. 2010;49(3):189-206.

3. Li GF, Wang K, Chen R, Zhao HR, Yang J, Zheng QS. Simulation of the pharmacokinetics of bisoprolol in healthy adults and patients with impaired renal function using wholebody physiologically based pharmacokinetic modeling. Acta Pharmacol Sin. 2012;33(11):1359-71.

4. Vogt W. Evaluation and optimisation of current milrinone prescribing for the treatment and prevention of low cardiac output syndrome in paediatric patients after open heart surgery using a physiology-based pharmacokinetic drug-disease model. Clin Pharmacokinet. 2014;53(1):51-72.

5. Sayama H, Takubo H, Komura H, Kogayu M, Iwaki M. Application of a physiologically based pharmacokinetic model informed by a top-down approach for the prediction of pharmacokinetics in chronic kidney disease patients. AAPS J. 2014;16(5):1018-28.

6. Edginton AN, Willmann S. Physiology-based simulations of a pathological condition: prediction of pharmacokinetics in patients with liver cirrhosis. Clin Pharmacokinet. 2008;47(11):743-52.

7. Khalil F, Laer S. Physiologically based pharmacokinetic modeling: methodology, applications, and limitations with a focus on its role in pediatric drug development. J Biomed Biotechnol. 2011;2011:907461.

8. Rowland Yeo K, Aarabi M, Jamei M, Rostami-Hodjegan A. Modeling and predicting drug pharmacokinetics in patients with renal impairment. Expert Rev Clin Pharmacol. 2011;4(2):261-74.

9. Leithe ME, Margorien RD, Hermiller JB, Unverferth DV, Leier CV. Relationship between central hemodynamics and regional blood flow in normal subjects and in patients with congestive heart failure. Circulation. 1984;69(1):57-64.

10. Nies AS, Shand DG, Wilkinson GR. Altered hepatic blood flow and drug disposition. Clin Pharmacokinet. 1976;1(2):135-55.

11. Abdelaziz A, al-Araby M, Mahran L, Spahn-Langguth H. Active metabolites formed during hepatic first-pass: simulations featuring their contribution to the overall effect in altered liver clearance and drug-drug interactions. BMC Pharmacol. 2009;9(Suppl 2):A38.

12. Neugebauer G, Akpan W, Kaufmann B, Reiff K. Stereoselective disposition of carvedilol in man after intravenous and oral administration of the racemic compound. Eur J Clin Pharmacol. 1990;38(2):S108-11.

13. Neugebauer G, Akpan W, von Mollendorff E, Neubert P, Reiff K. Pharmacokinetics and disposition of carvedilol in humans. J Cardiovasc Pharmacol. 1987;10(Suppl 11):S85-8.

14. Neugebauer G, Neubert P. Metabolism of carvedilol in man. Eur J Drug Metab Pharmacokinet. 1991;16(4):257-60.

15. Behn F. Pharmakokinetik, Pharmakodynamik und Pharmakogenetik von Carvedilol in Abhängigkeit vom Lebensalter bei pädiatrischen Patienten mit Herzinsuffizienz. Dissertation zur Erlangung des Doktorgrades des Fachbereichs Chemie der Universität Hamburg; 2001.

16. Tenero D, Boike S, Boyle D, Ilson B, Fesniak HF, Brozena S, et al. Steady-state pharmacokinetics of carvedilol and its enantiomers in patients with congestive heart failure. J Clin Pharmacol. 2000;40(8):844-53.

17. Giessmann T, Modess C, Hecker U, Zschiesche M, Dazert P, Kunert-Keil C, et al. CYP2D6 genotype and induction of intestinal drug transporters by rifampin predict presystemic clearance of carvedilol in healthy subjects. Clin Pharmacol Ther. 2004; 75(3):213-22.

18. von Mollendorff E, Reiff K, Neugebauer G. Pharmacokinetics and bioavailability of carvedilol, a vasodilating beta-blocker. Eur J Clin Pharmacol. 1987;33(5):511-3.

19. Jamei M, Marciniak S, Feng K, Barnett A, Tucker G, RostamiHodjegan A. The Simcyp population-based ADME simulator. Expert Opin Drug Metab Toxicol. 2009;5(2):211-23.

20. Khalil F, Laer S. Physiologically based pharmacokinetic models in the prediction of oral drug exposure over the entire pediatric age range-sotalol as a model drug. AAPS J. 2014;16(2):226-39.

21. Jamei M, Turner D, Yang J, Neuhoff S, Polak S, Rostami-Hodjegan A, et al. Population-based mechanistic prediction of oral drug absorption. AAPS J. 2009;11(2):225-37.

22. Bachmakov I, Werner U, Endress B, Auge D, Fromm MF. Characterization of beta-adrenoceptor antagonists as substrates 
and inhibitors of the drug transporter P-glycoprotein. Fundam Clin Pharmacol. 2006;20(3):273-82.

23. Davis SS, Hardy JG, Fara JW. Transit of pharmaceutical dosage forms through the small intestine. Gut. 1986;27(8):886-92.

24. Kaijser M, Johnsson C, Zezina L, Backman U, Dimeny E, Fellstrom B. Elevation of cyclosporin A blood levels during carvedilol treatment in renal transplant patients. Clin Transplant. 1997;11(6):577-81.

25. Giacomini KM, Huang SM, Tweedie DJ, Benet LZ, Brouwer KL, Chu X, et al. Membrane transporters in drug development. Nat Rev Drug Discov. 2010;9(3):215-36.

26. Wessler JD, Grip LT, Mendell J, Giugliano RP. The P-glycoprotein transport system and cardiovascular drugs. J Am Coll Cardiol. 2013;61(25):2495-502.

27. Berezhkovskiy LM. Volume of distribution at steady state for a linear pharmacokinetic system with peripheral elimination. J Pharm Sci. 2004;93(6):1628-40.

28. Kilford PJ, Stringer R, Sohal B, Houston JB, Galetin A. Prediction of drug clearance by glucuronidation from in vitro data: use of combined cytochrome P450 and UDP-glucuronosyltransferase cofactors in alamethicin-activated human liver microsomes. Drug Metab Dispos. 2009;37(1):82-9.

29. Hanioka N, Tanaka S, Moriguchi Y, Narimatsu S. Stereoselective glucuronidation of carvedilol in human liver and intestinal microsomes. Pharmacology. 2012;90(3-4):117-24.

30. Salem F, Johnson TN, Abduljalil K, Tucker GT, Rostami-Hodjegan A. A re-evaluation and validation of ontogeny functions for cytochrome P450 $1 \mathrm{~A} 2$ and $3 \mathrm{~A} 4$ based on in vivo data. Clin Pharmacokinet. 2014;53(7):625-36.

31. Cubitt HE, Yeo KR, Howgate EM, Rostami-Hodjegan A, Barter ZE. Sources of interindividual variability in IVIVE of clearance: an investigation into the prediction of benzodiazepine clearance using a mechanistic population-based pharmacokinetic model. Xenobiotica. 2011;41(8):623-38.

32. Oldham HG, Clarke SE. In vitro identification of the human cytochrome P450 enzymes involved in the metabolism of $\mathrm{R}(+)$ and S(-)-carvedilol. Drug Metab Dispos. 1997;25(8):970-7.

33. Sehrt D, Meineke I, Tzvetkov M, Gultepe S, Brockmoller J. Carvedilol pharmacokinetics and pharmacodynamics in relation to CYP2D6 and ADRB pharmacogenetics. Pharmacogenomics. 2011;12(6):783-95.

34. Gehr TW, Tenero DM, Boyle DA, Qian Y, Sica DA, Shusterman $\mathrm{NH}$. The pharmacokinetics of carvedilol and its metabolites after single and multiple dose oral administration in patients with hypertension and renal insufficiency. Eur J Clin Pharmacol. 1999;55(4):269-77.

35. Wilkinson GR, Shand DG. Commentary: a physiological approach to hepatic drug clearance. Clin Pharmacol Ther. 1975;18(4):377-90.

36. Johnson TN, Rostami-Hodjegan A. Resurgence in the use of physiologically based pharmacokinetic models in pediatric clinical pharmacology: parallel shift in incorporating the knowledge of biological elements and increased applicability to drug development and clinical practice. Paediatr Anaesth. 2011;21(3):291-301.

37. Salem F, Johnson TN, Barter ZE, Leeder JS, Rostami-Hodjegan A. Age related changes in fractional elimination pathways for drugs: assessing the impact of variable ontogeny on metabolic drug-drug interactions. J Clin Pharmacol. 2013;53(8):857-65.

38. Laer S, Mir TS, Behn F, Eiselt M, Scholz H, Venzke A, et al. Carvedilol therapy in pediatric patients with congestive heart failure: a study investigating clinical and pharmacokinetic parameters. Am Heart J. 2002;143(5):916-22.

39. The Criteria Committee of the New York Heart Association. Nomenclature and criteria for diagnosis of diseases of the heart and great vessels. 9th edn. Boston: Little, Brown and Company; 1994.

40. Ross RD, Bollinger RO, Pinsky WW. Grading the severity of congestive heart failure in infants. Pediatr Cardiol. 1992;13(2):72-5.

41. Marsousi N, Daali Y, Rudaz S, Almond L, Humphries H, Desmeules $\mathrm{J}$, et al. Prediction of metabolic interactions with oxycodone via CYP2D6 and CYP3A inhibition using a physiologically based pharmacokinetic model. CPT Pharmacomet Syst Pharmacol. 2014;3(12):1-8.

42. Jiang XL, Zhao P, Barrett JS, Lesko LJ, Schmidt S. Application of physiologically based pharmacokinetic modeling to predict acetaminophen metabolism and pharmacokinetics in children. CPT Pharmacomet Syst Pharmacol. 2013;2:e80.

43. Jamei M, Bajot F, Neuhoff S, Barter Z, Yang J, Rostami-Hodjegan $\mathrm{A}$, et al. A mechanistic framework for in vitro-in vivo extrapolation of liver membrane transporters: prediction of drugdrug interaction between rosuvastatin and cyclosporine. Clin Pharmacokinet. 2014;53(1):73-87.

44. Parrott N, Paquereau N, Coassolo P, Lave T. An evaluation of the utility of physiologically based models of pharmacokinetics in early drug discovery. J Pharm Sci. 2005;94(10):2327-43.

45. Johnson TN, Rostami-Hodjegan A, Tucker GT. Prediction of the clearance of eleven drugs and associated variability in neonates, infants and children. Clin Pharmacokinet. 2006;45(9):931-56.

46. Jones HM, Parrott N, Jorga K, Lave T. A novel strategy for physiologically based predictions of human pharmacokinetics. Clin Pharmacokinet. 2006;45(5):511-42.

47. De Buck SS, Sinha VK, Fenu LA, Nijsen MJ, Mackie CE, Gilissen RA. Prediction of human pharmacokinetics using physiologically based modeling: a retrospective analysis of 26 clinically tested drugs. Drug Metab Dispos. 2007;35(10):1766-80.

48. Gertz M, Houston JB, Galetin A. Physiologically based pharmacokinetic modeling of intestinal first-pass metabolism of CYP3A substrates with high intestinal extraction. Drug Metab Dispos. 2011;39(9):1633-42.

49. Abduljalil K, Cain T, Humphries H, Rostami-Hodjegan A. Deciding on success criteria for predictability of pharmacokinetic parameters from in vitro studies: an analysis based on in vivo observations. Drug Metab Dispos. 2014;42(9):1478-84.

50. Ross RD. The Ross classification for heart failure in children after 25 years: a review and an age-stratified revision. Pediatr Cardiol. 2012;33(8):1295-300.

51. Fleming S, Thompson M, Stevens R, Heneghan C, Pluddemann A, Maconochie I, et al. Normal ranges of heart rate and respiratory rate in children from birth to 18 years of age: a systematic review of observational studies. Lancet. 2011;377(9770):1011-8.

52. Tanaka H, Monahan KD, Seals DR. Age-predicted maximal heart rate revisited. J Am Coll Cardiol. 2001;37(1):153-6.

53. Noda T, Todani T, Watanabe Y, Yamamoto S. Liver volume in children measured by computed tomography. Pediatr Radiol. 1997;27(3):250-2.

54. Anderson GD. Developmental pharmacokinetics. Semin Pediatr Neurol. 2010;17(4):208-13.

55. Caron G, Steyaert G, Pagliara A, Reymond F, Crivori P, Gaillard $\mathrm{P}$, et al. Structure-lipophilicity relationships of neutral and protonated $\beta$-blockers, part I, intra- and intermolecular effects in isotropic solvent systems. Helvetica Chimica Acta. 1999;82(8):1211-22.

56. Fujimaki M, Murakoshi Y, Hakusui H. Assay and disposition of carvedilol enantiomers in humans and monkeys: evidence of stereoselective presystemic metabolism. J Pharm Sci. 1990;79(7):568-72. 